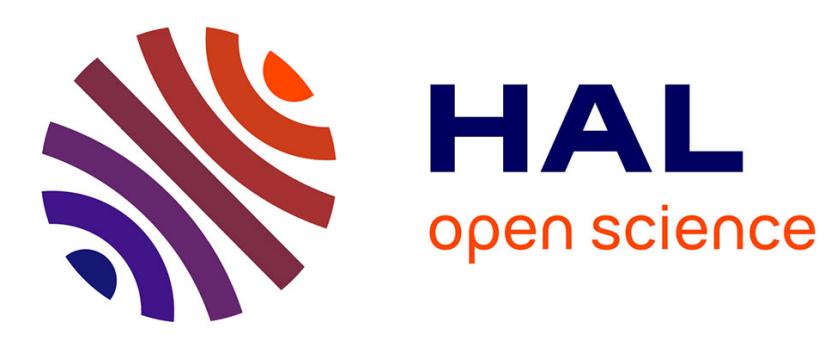

\title{
Water retention against drying with soft-particle suspensions in porous media
}

Emmanuel Keita, Thomas E. Kodger, Pamela Françoise Faure, Stéphane Rodts, David A. Weitz, Philippe Coussot

\section{- To cite this version:}

Emmanuel Keita, Thomas E. Kodger, Pamela Françoise Faure, Stéphane Rodts, David A. Weitz, et al.. Water retention against drying with soft-particle suspensions in porous media. Physical Review E , 2016, 94 (3), 10.1103/PhysRevE.94.033104 . hal-02136443

\section{HAL Id: hal-02136443 \\ https://hal-enpc.archives-ouvertes.fr/hal-02136443}

Submitted on 22 May 2019

HAL is a multi-disciplinary open access archive for the deposit and dissemination of scientific research documents, whether they are published or not. The documents may come from teaching and research institutions in France or abroad, or from public or private research centers.
L'archive ouverte pluridisciplinaire HAL, est destinée au dépôt et à la diffusion de documents scientifiques de niveau recherche, publiés ou non, émanant des établissements d'enseignement et de recherche français ou étrangers, des laboratoires publics ou privés. 


\title{
Water retention against drying with soft particle suspensions in porous media
}

\author{
E. Keita ${ }^{a, b}$, T. E. Kodger ${ }^{a}$, P. Faure ${ }^{\text {b }}$, S. Rodts ${ }^{\text {b }}$, D. A. Weitz ${ }^{a}$, P. Coussot ${ }^{b}$ \\ a Experimental Soft Matter Group, Harvard University, Cambridge, Massachusetts 02138, USA \\ ${ }^{\mathrm{b}}$ Université Paris-Est, Laboratoire Navier, ENPC-IFSTTAR-CNRS, Champs-sur-Marne, France
}

\begin{abstract}
Polymers suspended in granular packings have a significant impact on water retention, which is important for soil irrigation and the curing of building materials. Whereas the drying rate remains constant during a long period for pure water due to capillary flow providing liquid water to the evaporating surface, we show that it is not the case for a suspension made of soft polymeric particles called microgels: the drying rate decreases immediately and significantly. By measuring the spatial water saturation and concentration of suspended particles with Magnetic Resonance Imaging (MRI), we can explain these original trends and model the process. In low viscous fluids, the accumulation of particles at the free surface induces a recession of the air-liquid interface. A simple model, assuming particle transport and accumulation below the sample free surface, is able to reproduce our observations without any fitting parameters. The high viscosity of the microgel suspension inhibits flow towards the free surface and a drying front appears. We show that water vapor diffusion over a defined and increasing length sets the drying rate. These results and model allow for better controlling the drying and water retention in granular porous materials.
\end{abstract}

\section{Introduction}

Many industrial processes aim to modify the liquid content in porous materials. In some cases, such as painting and food preservation, water must be removed from structures with mixed properties, such as softness, viscosity, and wettability [1]. Drying can be costly because it is slow or energy-intensive. However, in other cases, such as agriculture or the curing of concrete, fast drying is undesirable as trapping water improves the biological or chemical processes. Additives, such as colloids, polymers, hydrogels, and surfactants are used as irrigation water retention agents in soils $[2,3,4,5,6]$ and in mortars, mixed with the pasty material during the preparation $[7,8,9]$. It is likely that these agents tend to interact and form particulate systems which reduce water mobility [10], but the exact processes of these water retention effects are unclear. In particular, it has been observed that permeability is reduced when such agents are used [11], but there is little information about their exact role. As a consequence the dependence of evaporation on fluid composition remains poorly understood. This is partly due to lacking experimental tools capable of resolving opaque multiphasic porous medium. Improved measurements will provide insights in the relationship between microstructure and evaporation, which may benefit controlling liquid content of porous media and formulating efficient additives. 
Fundamental studies on the evaporation processes of porous media have mainly focused on pure water as the interstitial fluid. It was shown that, regardless the nature of the material structure, evaporation at the free surface leads to capillary pressure gradient and water flow. These mechanisms supply the free surface with liquid water and maintain a constant drying rate during most of the evaporation process $[12,13,14,15,16,17]$.

However water is rarely alone as an interstitial fluid. Salts formed by dissolution of reactive solids [18] or pollutants resulting from human activity are dispersed in soils [19] and may accumulate in some regions $[18,20,21,22]$ leading to the formation of crystals. Salt crystallization is of great concern for building materials [23] and drying kinetics show a strong dependence on the crystal structure [24, 25]. Additionally, construction materials or soils are made of particles in a wide range of sizes; the smallest particles tend to fill voids in granular packings increasing density and mechanical properties [26], but can be suspended and transported by the liquid. A recent study focused on the drying of dilute colloidal suspension initially saturating a model porous medium [27]. As interactions between them are low, the particles migrate towards the evaporative surface and form a deposit which limits further drying [27]. If interactions between particles are strong, a gel forms the interstitial fluid and the drying rate is strongly reduced [28].

These observations suggest that elements dispersed in water may significantly impact the drying rate. The present work addresses the drying of hydrogels. It is known that during drying, polymer solution drop exhibits transport and polymer deposit which limit water mobility [29]. However the processes at work can be complex depending on the element composition, interactions, and concentration. Hydrogels have the property that they can considerably deform or shrink during water extraction and thus are considered as soft, compressible particles distinguishable from hard particles [30].

In porous medium, drying of hydrogels is not just a question of physics related to the material properties but a complex coupling between boundary conditions (leading to a specific spatial distribution of vapor density) and the intrinsic properties of the polymer solution. To understand the behavior of hydrogels, here we focus on a model system, i.e. suspensions of microgels which are well controlled soft particles [31] exhibiting compaction behaviors set by their structure as water is removed [32], and compare the results with simpler systems, i.e. pure water and hard particles suspensions. Using Magnetic Resonance Imaging of Hydrogen $\left({ }^{1} \mathrm{H} \mathrm{MRI}\right)$ we are able to map the spatial water distribution inside the porous sample during drying. This information is used to deduce water flow leading to advection of microgels and a vapor diffusion length which sets the drying rate. Based on drying physics $[12,13,14]$, we explain the drying kinetics of a microgel suspension in a porous media using only bulk fluid properties, i.e. viscosity and surface tension.

\section{Materials and Methods}

We use a model porous medium: the solid undeformable structure is a granular skeleton filled by an interstitial liquid. The structure is made of $257(+/-9) \mu \mathrm{m}$ soda-lime glass beads packed by hand in a cylinder (height $H=4 \mathrm{~cm}$, diameter $=3.5 \mathrm{~cm}$ ) open on one side and filled with liquid. To avoid air bubbles, we vibrate constantly the sample while pouring gently the glass beads into the cylinder containing the liquid. The resulting packing fraction is $66 \%$ (based on mass measurements), therefore the porosity is $\omega=34 \%$ and the mean pore radius is $r \approx 38 \mu \mathrm{m}$ (computed from X-Ray microtomography [33]), which fairly corresponds to commonly admitted value of one sixth of bead diameter. 
The soft particles are homemade microgel particles which are micrometer hydrogel sponges composed of poly( $\mathrm{N}$-isopropylacrylamide-co-acrylic acid) [31, 32]. These soft particles are synthesized by precipitation polymerization mixing NIPAm ( $\mathrm{N}$-isopropyl acrylamide, Tokyo Chemical Industries), acrylic acid (AAc, Sigma), N,N-methylenebisacrylamide (BIS, Sigma) and synthesized fluorescent cores (diameter $160 \mathrm{~nm}$ ) composed of perfluoroethyl-methacrylate (PFEMA, dyed with Nile red) in $300 \mathrm{~mL}$ of deionized water. The amount of the crosslinker, BIS, is $1 \mathrm{wt} \%$ relative to NIPAm monomer. The reaction temperature is maintained at $70^{\circ} \mathrm{C}$ and the fluid is continuously mixed and bubbled with Argon for $10 \mathrm{~min}$ to remove oxygen which inhibits free radicals. Then we add potassium persulfate (KPS, Sigma) as an initiator. After 2 hours the reaction is filtered with filter paper (pores of $30 \mu \mathrm{m}$ ) to remove large aggregates and cooled. The total reaction conversion is typically $95 \%$, and the suspension is cleaned of unreacted monomer by repeated centrifugation and dispersion in a buffer solution of HEPES (100 mM, acid dissociation constant $p K a=7.5$, from Sigma). The concentration of AAc within the microgel is estimated to be about $15 \mathrm{mM}$ with $p K a=4.25$, thus the buffer $p H$ dominates and AAc is fully ionized. Moreover, the buffer solution has a high ionic strength leading to purely repulsive short range interactions between particles.

(a)

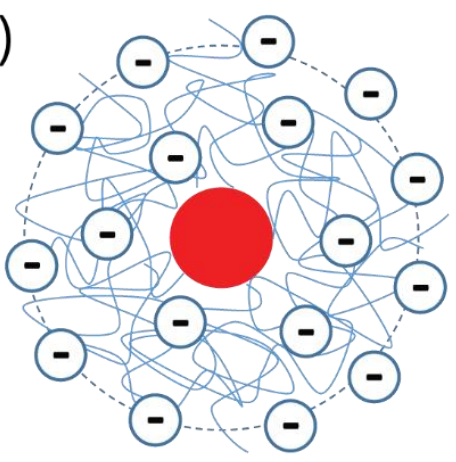

(b)

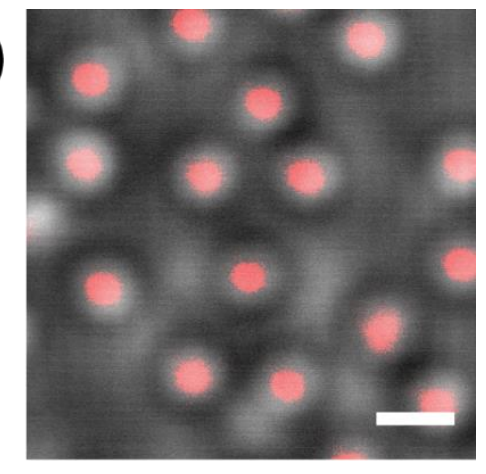

Figure 1: (a) Schematic of a microgel particle composed of a rigid fluorescent core $(160 \mathrm{~nm})$ and a pNIPAM structure with negative charges at $\mathrm{pH}>4.25$ ( $\mathrm{pKa}$ of acrylic acid is 4.25). Charges are throughout the structure; polymer chains extend at the surface. (b) Image of a microgel suspension (superposition of fluorescent and bright field images). The scale bar is $1 \mu \mathrm{m}$.

Microgels are polymer networks swollen with water of micro-scale dimensions in dilute solution. From the microscope image of a microgel suspension on a glass slide, we see the fluorescence of the red cores and the particle shapes. The microgel particles are spherical and fairly monodisperse with a radius slightly larger than $1 \mu \mathrm{m}$. Each particle has a red core which appears larger than its actual size (see Figure 1). From Dynamic Light Scattering measurements we measure the radius to be around $1 \mu \mathrm{m}$ at $20^{\circ} \mathrm{C}$ and $0.2 \mu \mathrm{m}$ at $40^{\circ} \mathrm{C}$ (above the pNIPAm lower critical solution temperature, LCST $\approx 31^{\circ} \mathrm{C}$ ) emphasizing the compressibility of the particles. Thus microgels are homogenous, spherical and compressible particles.

The microgel concentration is measured by dry extract content and suspension viscosity at low shear rate in an Anton Paar MCR501 rheometer with a Double-Couette geometry. For dilute suspensions ( $c<5 \mathrm{~g} / \mathrm{L}$ ), the relative viscosity $\eta_{r}$, the ratio of the suspension to pure water viscosity, remains below 5 (see Figure 2). For concentrated suspensions, the particles touch each other, deform to have flat facets [34], and reduce their volume. The suspension is still fluid but $\eta_{r}$ increases from 5 to 30 for $c \approx 10-50 \mathrm{~g} / \mathrm{L}$ (see Figure 2). As there is no interstitial water left between particles ( $c_{m} \approx 30 \mathrm{~g} / \mathrm{L}$ ), they 
compress. To flow, particles have to slide along one another but they finally form a jammed structure and a yield stress appears around $c_{\text {yield }} \approx 80 \mathrm{~g} / \mathrm{L}$. The flow of concentrated microgels has been studied in [32].

We use three microgel suspensions varying the microgel initial concentration as $c_{0}=3.5-10.4-45.1$ $\mathrm{g} / \mathrm{L}$ to have initial fluid suspensions and represent the different regimes: dilute, interparticle contacts, and compressed particles.

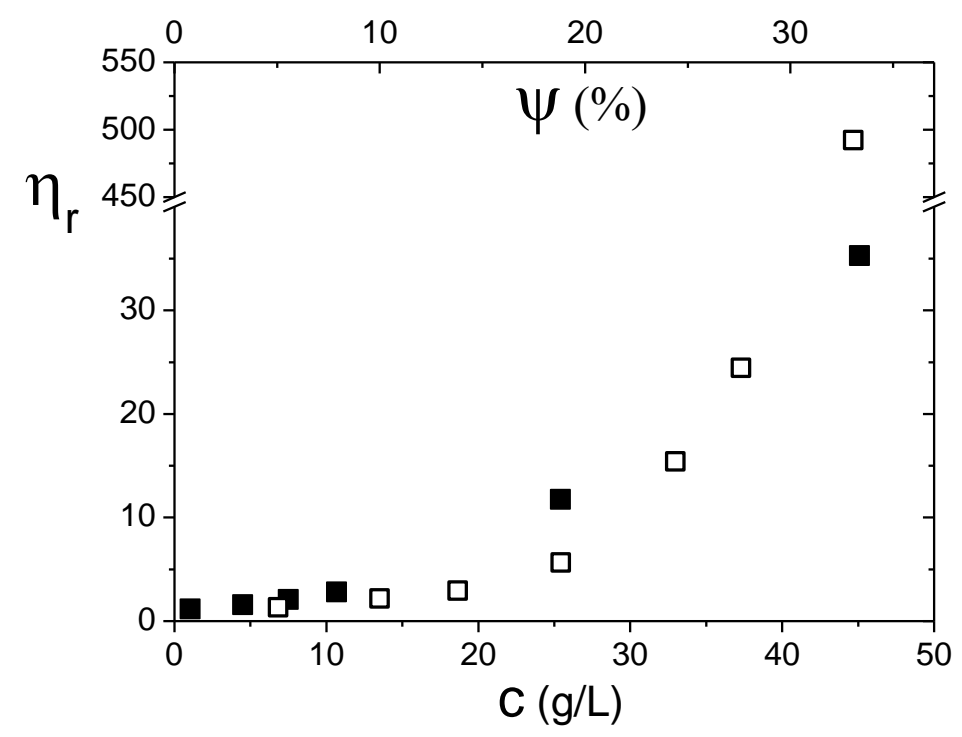

Figure 2: Relative viscosity of silica suspension in water (open squares) as a function of the volume concentration of particles (top abscissa), and of microgel suspension (filled squares) as a function of the microgel mass concentration (bottom abscissa).

Additionally, we use silica nanoparticles (Ludox HS-40, diameter $d=20 \mathrm{~nm}$, particles density $\approx 2.45$ $\mathrm{g} / \mathrm{mL}$ ) as hard particles. They are not deformable and are stabilized by electrostatic repulsion due to negative charges on the surface at $p H=9.8$. Silica particle suspensions are Newtonian fluids with a viscosity increasing with the volume fraction $(\psi)$ from that of water for $\psi<5 \%$ to a relative viscosity (suspension to water viscosity ratio) $\eta_{r} \approx 500$ for $\psi=33 \%$ (see Figure 2). This is in good agreement with rheological studies on similar suspensions [34] which report a sharp increase of viscosity around $\psi \approx 30 \%$. At higher volume fraction, the suspension is not Newtonian but has a yield stress to overcome before it starts to flow. The maximum random packing fraction is estimated to be in the range $\psi=58-64 \%[34,35]$. We varied the initial volume fraction of silica suspension from $\psi_{0}=5$ to $33 \%$.

Each sample dries in the Magnetic Resonance Imaging (MRI) apparatus with a dried air flux (Relative Humidity $<1 \%$, room temperature $T=25^{\circ} \mathrm{C}$ ) controlled by a flow meter to maintain constant external conditions. The air flow has an average air velocity of $0.1 \mathrm{~m} / \mathrm{s}$ above the sample free surface in a confined channel (section: $6 \mathrm{~cm} \times 1 \mathrm{~cm}$ ), the sample is level with the channel bottom surface (see Figure 3). MRI experiments are performed in a Bruker 24/80 DBX spectrometer operating at 0.5T (20MHz proton frequency), with a $20 \mathrm{~cm}$ wide measuring area allowing the insertion of the full drying setup. 
Measurements only observe hydrogen atoms in the sample and, in the present case, are almost exclusively sensitive to that of water molecules.

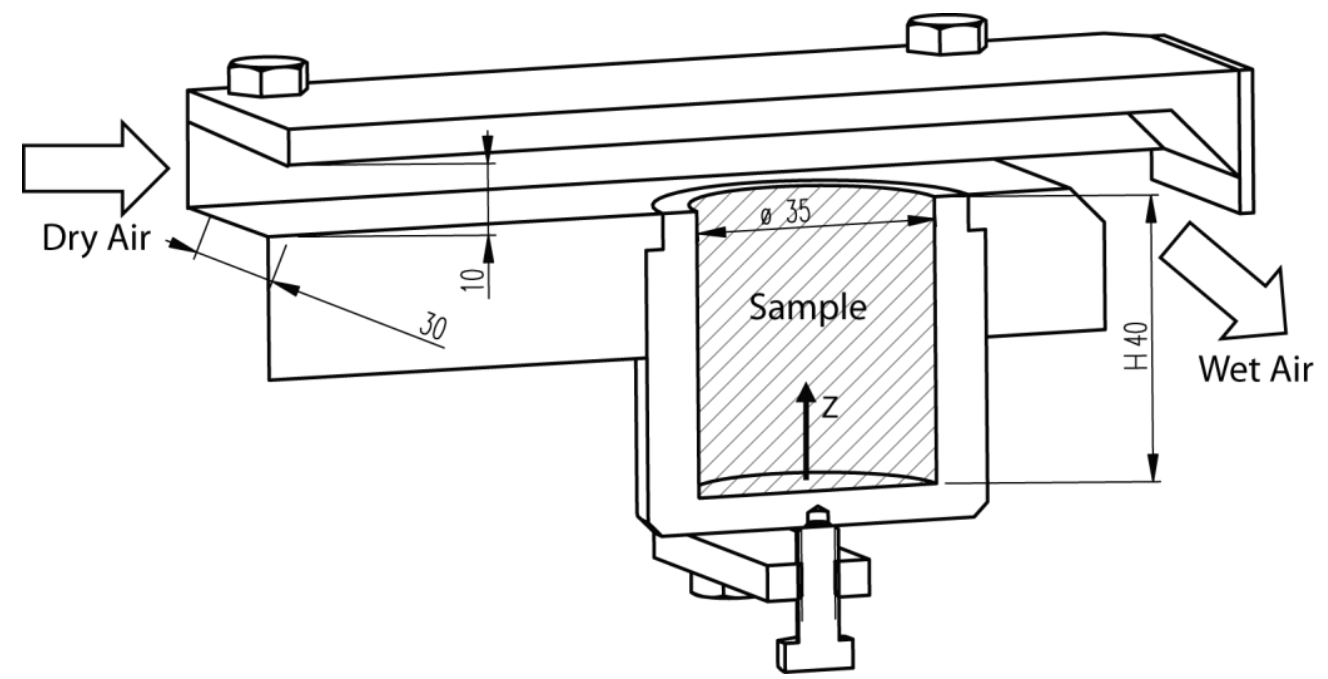

Figure 3: Design of the drying set-up in the MRI. The sample is a cylinder ( $\mathrm{H}=40 \mathrm{~mm}$, diameter $=35 \mathrm{~mm})$. Its upper surface is in contact with a $10 \mathrm{~mm}$ high channel where dry air flow at constant flux. For imaging, MRI water signal is measured along the z-axis of the sample.

A set of measurements (total water mass, water distribution, relaxation time profile) is performed every 2 hours with an acquisition time kept below 1 hour, similar to the procedure described in [27]. In the case of silica suspensions the particle distribution could also be measured. Below we describe the whole procedure and processing providing the water and particle distributions in more details.

As the sample stays during the whole experiment in the MRI set-up, we measure the total water mass by the intensity of the overall Nuclear Magnetic Resonance (NMR) signal emitted by the sample in a Free Induction Decay experiment. Both quantities are indeed proportional to each other. Measuring the initial mass $\left(m_{i n i}\right)$ with a balance prior to experiment provides the necessary calibration for monitoring water mass in the sample $(m)$ as a function of time. We define the water content $\left(\mathrm{m} / \mathrm{m}_{i n i}\right)$ and the global water saturation $\phi$, as the ratio of water to pore volume. Dried microgels occupy a small volume therefore $\phi \approx m / m_{i n i}$, but silica particles occupy a significant part of the pore volume: $\phi=\left(1-\psi_{0}\right) \mathrm{m} / \mathrm{m}_{\text {ini }}$.

The distribution of apparent water along $z$ axis of the sample is measured with a one-dimensional double spin-echo sequence (two first echoes of the so-called Carr-Purcell-Meibom-Gill sequence [36, 37] with added imaging gradients and exponential extrapolation to compensate spin-spin relaxation). Each measured value of this distribution corresponds to the total amount of water in a thin crosssectional layer $(300 \mu \mathrm{m})$ situated at a given position along the sample axis. Data values under 4 times the standard deviation of the noise were disregarded to avoid aberration in the post processing. Thus the wetted portion of the sample can be identified and gives a clear position of the dried area (at $z=h$ ). Normalizing by the initial value, we obtain the local water saturation $\phi(z)$ i.e. the local ratio of water to pore volume. 
Combining water distribution with relaxation time profile, we calculate the particle distribution $\chi$ (ratio of particles to pore volume) and the particle concentration, i.e. the ratio of particle to fluid volume $\psi=\chi /(\chi+\phi)$. Water spin-lattice relaxation time $\mathrm{T}_{1}$ is computed from series of $\mathrm{T}_{1}$-weighted spin-echo profiles. During this sequence, the signal $s$ evolves as follows [38]:

$$
s(t)-s(t \rightarrow \infty)=-2 \cdot m \cdot \exp \left(-\frac{t}{T_{1}}\right)
$$

where $t$ is the time after excitation. In order to avoid excessive measuring time, $T_{1}$ weighted profiles were obtained for a restricted list of 5 values of $t$ delay, and further fitted for each position by means of eq. 1 to determine local $T_{1}$ values. The delay list was optimized so as to get accurate estimates of $T_{1}$ over the full range of values expected to be explored during sample drying.

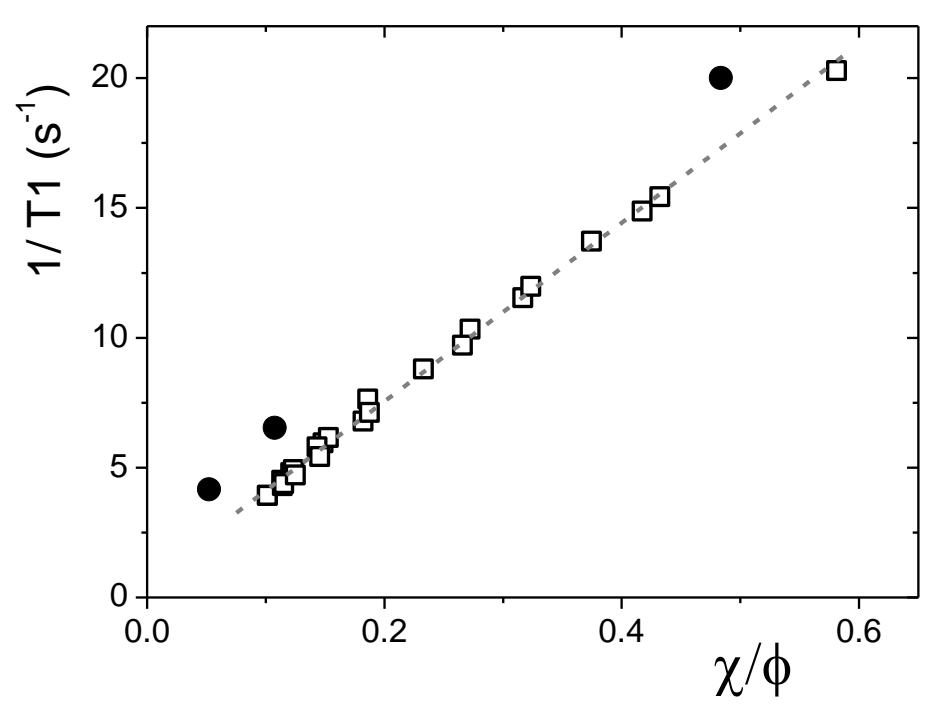

Figure 4 : NMR relaxation in silica particles suspensions. Measured 1/T1 values as a function of particle to water volume ratio (squares). Similar measurements for suspensions as interstitial fluid in a saturated bead packing (circles). The grey line is the linear fit of equation (2) for suspensions with $\alpha=35.2 \mathrm{~s}^{-1}$

As compared with free water (for which $T_{1, b u l k}=2.5 \mathrm{~s}$ ), the relaxation time established from (1) decreases when water gets into contact with solids. As in the case of clay [39] and cement [40] suspensions, experiments on stable silica suspensions indicate that relaxation $1 / T_{1}$ depends fairly linearly on particle to water volume ratio over a wide range of particle concentrations (see Figure 4) in good agreement with the theoretical frame of the fast exchange assumption [36, 37, 41]:

$$
\frac{1}{T_{1}}=\frac{1}{T_{1, b u l k}}+\alpha \frac{\chi}{\phi}
$$

where $\alpha=35.2 \mathrm{~s}^{-1}$ depends on specific area and particle relaxation properties (see Figure 4). Specific area of glass beads is smaller compared to that of silica particles but nevertheless induces a clear increase of $1 / T_{1}$ for granular packing filled with silica particle suspension (see Figure 4 ). The specific 
contribution of glass beads to $1 / T_{1}$ is calibrated with a drying experiment where pure water is used as the interstitial fluid (not shown here). Relaxation enhancement due to water contact with solid surfaces of the porous matrix is empirically fitted with saturation while drying:

$$
\rho(\phi)=\frac{1}{T_{1}}-\frac{1}{T_{1, \text { bulk }}}=\frac{1}{\omega \phi}\left(0.65-\frac{0.065}{\omega \phi+0.1}\right)
$$

In presence of multiple solid surfaces, and within the frame of the fast exchange assumption, which was checked to be valid in our case, various relaxation contribution simply add together [36, 37, 41]. Assuming that the repartition of the suspension is similar to pure water, and granted that in (3) the first and second occurrence of $\omega \phi$ should be physically interpreted as water (without particles) and fluid (with particles) content respectively, the relaxation time in a packing of glass beads partially saturated with a particle suspension finally reads:

$$
\frac{1}{T_{1}}=\frac{1}{T_{1, \text { bulk }}}+\frac{1}{\omega \phi}\left(0.65-\frac{0.065}{\omega(\phi+\chi)+0.1}\right)+\alpha \frac{\chi}{\phi}
$$

Considering both $T_{1}$ and $\phi$ as raw information provided by MRI, the particle distribution $\chi$ then turns out to be the solution of a quadratic equation. In the case of microgels, above NMR technique could however not be used. Relaxation parameter $\alpha$ indeed turned out too low to keep NMR sensitivity to soft particles.

\section{Results}

\section{a. Macroscopic observations}

Macroscopically, drying is measured by mass loss corresponding to the evaporation of liquid water. For pure water as interstitial fluid, the mass loss is fairly linear with time over most of the drying duration and the sample is completely dry after 72 hours (see Figure 5.a). For microgel suspensions, evaporation is slower than for pure water, suggesting an impact of the particles on the drying kinetics. Moreover the mass loss decreases with increasing microgel concentration. The initial water mass is approximately the same for all samples and at the end of experiments, the samples with microgels are still partially saturated with water. For low microgel concentration $\left(c_{0}=3.5 \mathrm{~g} / \mathrm{L}\right)$, the mass loss is comparable to pure water in the first 10 hours but at the end of the experiment, after 50 hours, it is significantly lower with only $60 \%$ of the water evaporated. For higher concentrations, the mass loss is lower and the difference in drying kinetics can be observed at earlier times. Less than a third of the water available evaporates in 4 days. While for pure water the mass loss is proportional to $t$ over most of the evaporation process; for high concentration of microgels, the mass loss scales with $\sqrt{t}$ (see Figure 5.b).

The drying rate is computed as the mass loss per unit of time. For pure water, after an initial decrease, the drying rate is constant around $0.3 \mathrm{~g} / \mathrm{h}$ until $25 \%$ of the water remains. Then the drying rate decreases sharply and is divided by a factor 10 (see Figure 5.c). The drying rate may depend on the sample surface or air flux conditions. In the following, in order to compare experiments as samples desaturate, the drying rate for all samples are normalized by the drying rate during the constant rate period for pure water $\left(d m / d t_{0} \approx 0.3 \mathrm{~g} / \mathrm{h}\right)$. 
For microgel suspensions, the drying rate is initially close to the one for pure water but it decreases for all microgel concentrations. For low microgel concentration, the drying rate decreases steadily and is around a third of the initial drying rate at $\mathrm{m} / \mathrm{m}_{i n i} \approx 0.4$. For higher concentrations, the drying rate decreases sharply by a factor around 4 as the saturation is still high.

(a)

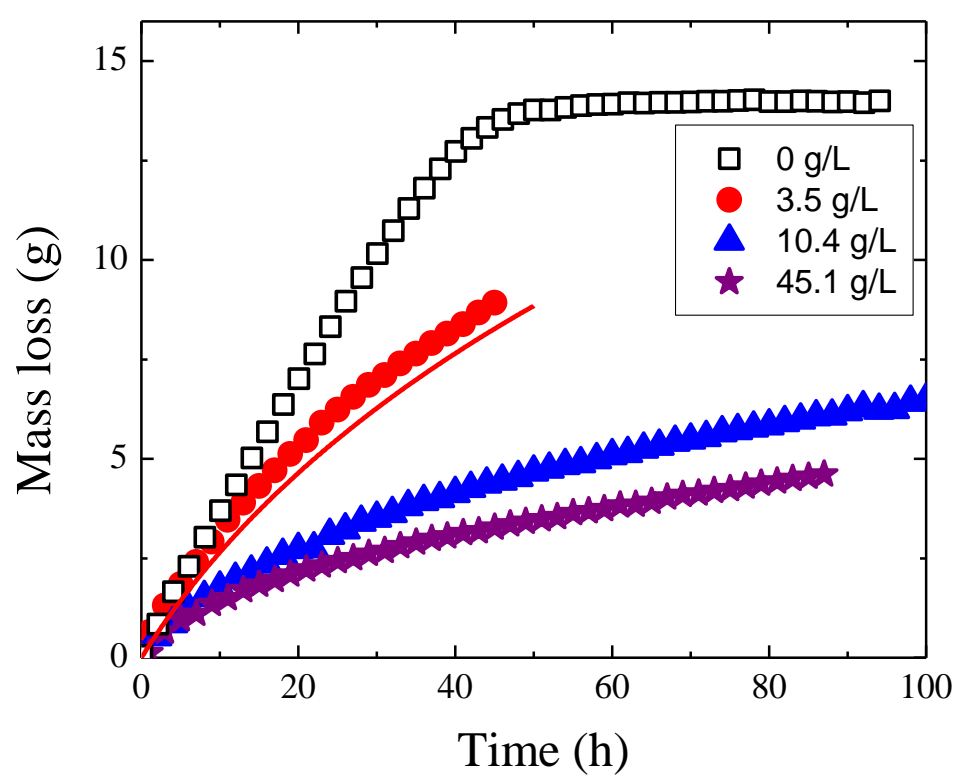

(b)

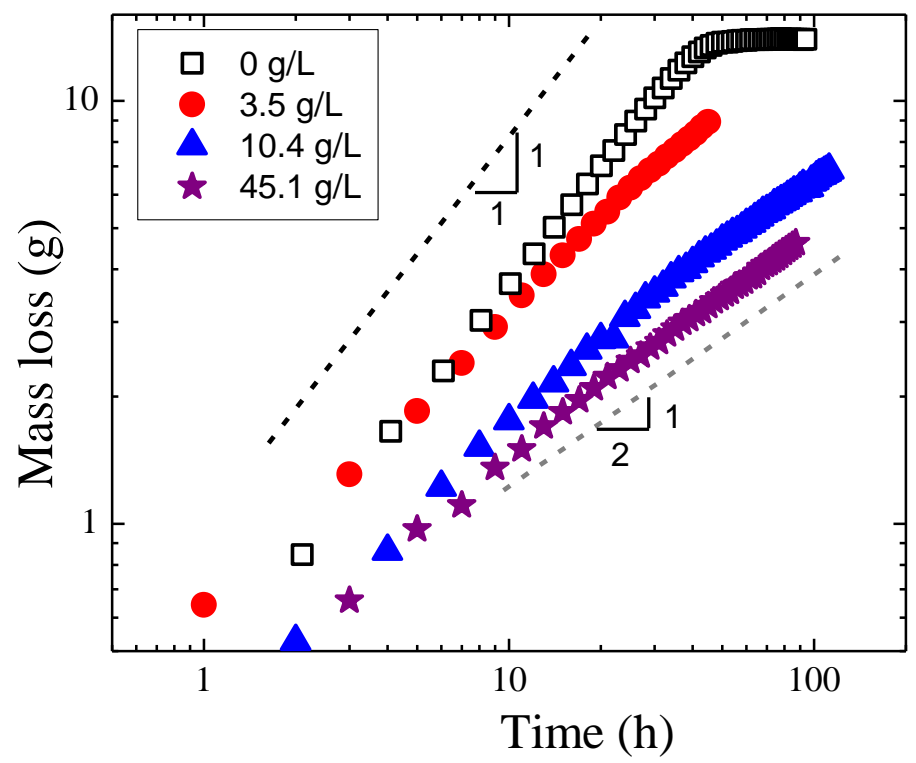


(c)

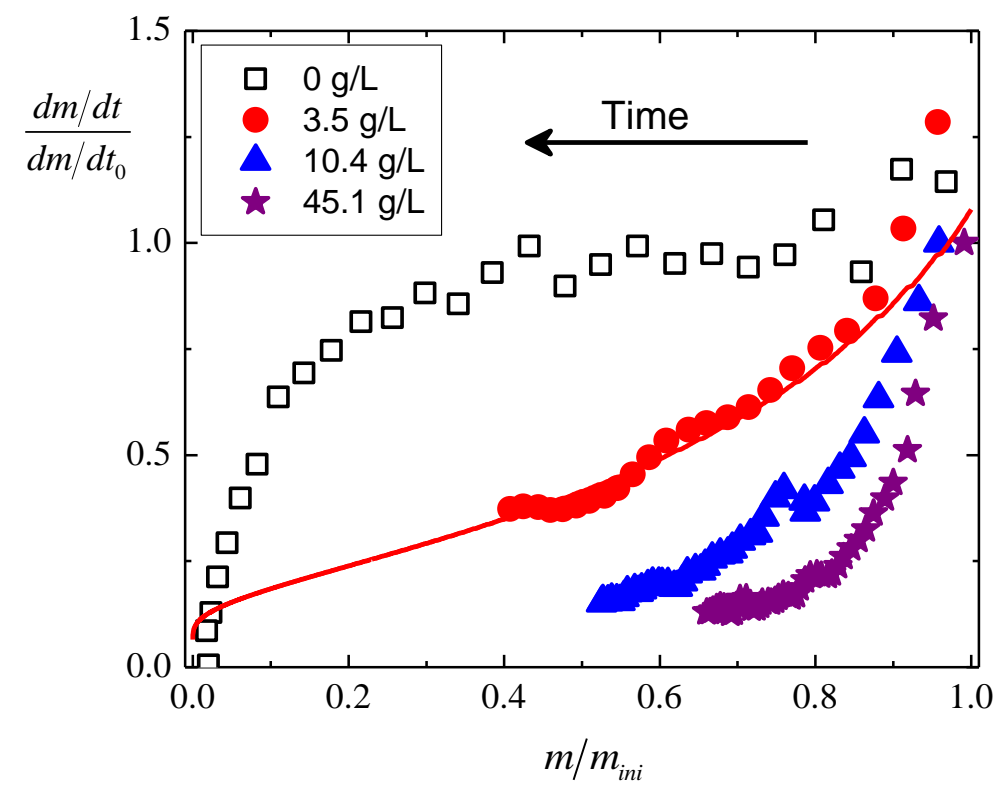

Figure 5: Drying of microgel suspensions in bead packing for different concentrations: pure water (squares), $c_{0}=3.5 \mathrm{~g} / \mathrm{L}$ (red circles), $10.4 \mathrm{~g} / \mathrm{L}$ (blue triangles), $45.1 \mathrm{~g} / \mathrm{L}$ (purple stars) and $33 \%$ (orange diamonds). Water mass loss as a function of time, in linear (a) and logarithmic scale (b). Drying rate normalized by initial value as a function of normalized water mass (c). The red continuous line corresponds to the migration-diffusion model for soft particles with $c_{0}=3.5 \mathrm{~g} / \mathrm{L}$.

For silica suspensions, evaporation is slower than for pure water (see Figure 6.a). As the initial volume fraction of silica particles increases from $\psi_{0}=5$ to $33 \%$, the mass loss decreases. The evaporated water decreases with volume fraction. Mass losses are similar during the first 10 hours then progressively evaporation slows down.

For silica suspensions at a volume fraction $\psi_{0}=5 \%$, the drying rate is fairly constant until $\mathrm{m} / \mathrm{m}_{i n i} \approx 0.7$ , then it decreases steadily to a tenth of its initial value (see Figure 6.b). For $\psi_{0}=10 \%$, the drying rate starts to decrease at $m / m_{i n i} \approx 0.8$. For higher volume fractions, the drying rate decreases constantly and reaches a minimum around a tenth of the initial drying rate at low saturation.

Crucially, the drying rate of water with the addition of silica particles gradually decreases, while for the addition of microgels, the drying rate decreases drastically. The effect of silica particles initially suspended in water is smoother than that observed with microgels (compare Figures 4 and 5): with silica suspensions the decrease in drying rate is quite progressive when the water content decreases whereas for microgels we observe a rapid decrease from the beginning of the drying process. 
(a)

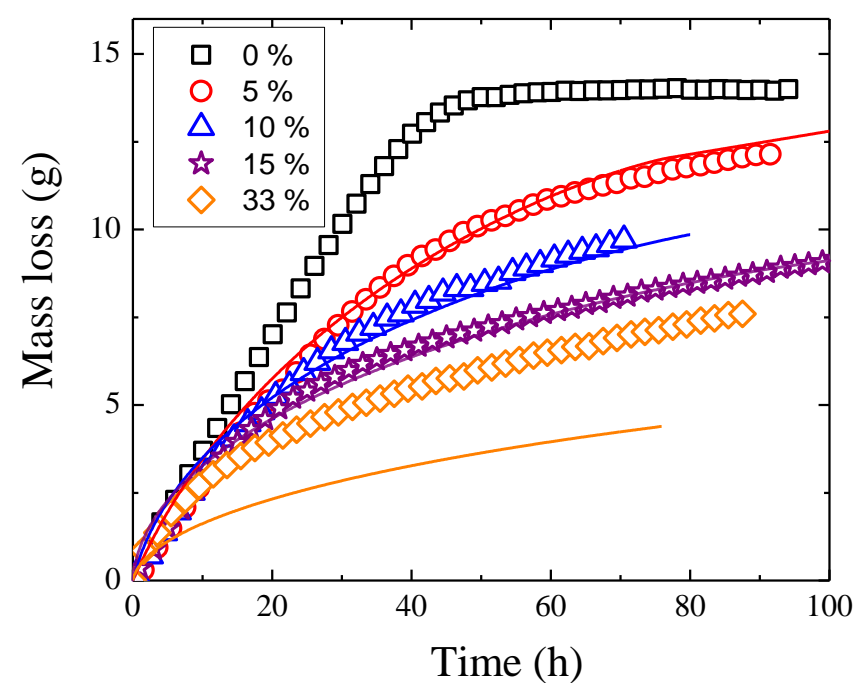

(b)

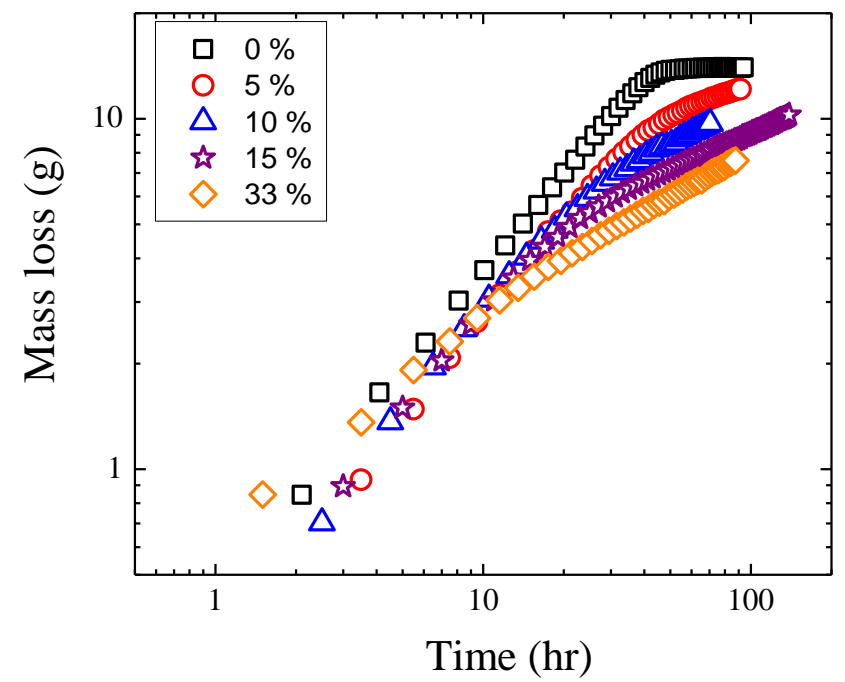

(c)

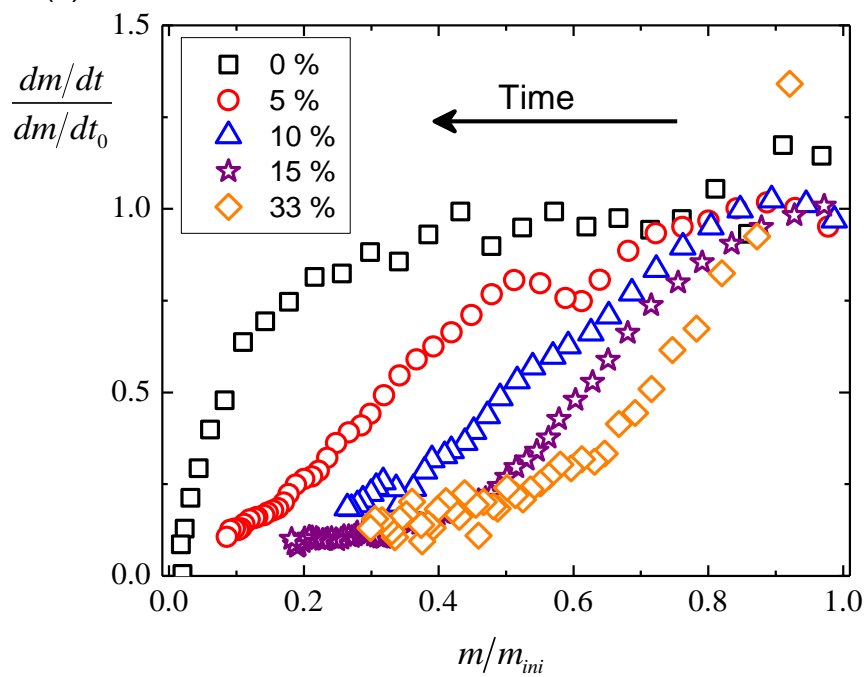


Figure 6: Drying of silica suspensions in bead packing for different concentrations: pure water (squares), $\psi_{0}=5 \%$ (red circles), 10\% (blue triangles), 15\% (purple stars) and 33\% (orange diamonds). Water mass loss as a function of time (a) and logarithmic scale (b). Drying rate normalized by initial value as a function of normalized water mass (c). The colored continuous lines correspond to the migration-diffusion model (see text).

\section{b. Local observations}

To differentiate the drying rate effects of microgels and silica particles, we measured the local water distribution by MRI in the porous media and calculate the local water saturation $\phi(z)$, the ratio of water to pore volume. Fluctuations of water content are observed at centimeter length scale (see for example around $z=3-3.5 \mathrm{~cm}$ in Figure 8.a). These distortions in the profiles are not deterministic but when they form, the next profiles are generally similar, i.e. the water amount decreases proportionally to the previous amount. Thus we consider that these fluctuations are due to packing heterogeneities and their positions and evolutions are not relevant within the frame of the analysis. It should be noted that the noise of the measurements is of the order of $d \phi \approx 6 \cdot 10^{-3}$. By contrast with the other regions and for the sake of simplicity, we consider that there is no water when the MRI signal is smaller than 4 times the noise level, and call the corresponding region, the "dry region". Initially we define the free surface as the first position for which water is observed yielding a criterion to define the length of the dry region below the free surface of the sample. Saturation decreases with time; we measured saturation profiles every 2 hours, but for the sake of clarity in the figures we present only a selection of profiles at different times. Evaporation occurs through the free surface at $z=4 \mathrm{~cm}$ as shown in Figure 6.

In the case of pure water filling the pores, the water saturation remains homogeneous down to low values (see Figure 7). The pores are initially filled with liquid, air enters progressively and reaches the bottom of the $4 \mathrm{~cm}$ sample after 16 hours. After which $\phi$ is homogenous at all heights, until $\phi<0.2$, when a dry front starts to invade the sample from the top but at the same time the saturation below goes on decreasing homogeneously. As explained above, the position $h$ of the dry front is defined as the last position with water signal above 4 times the standard deviation of the noise in our MRI profile. The corresponding dry area of size $h$ increases slowly to $h \approx 4 \mathrm{~mm}$ until $\phi \approx 0.05$.

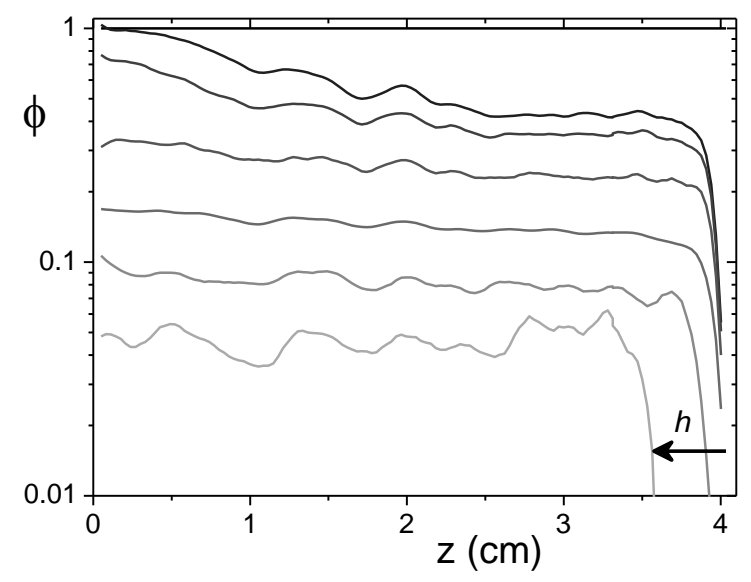

Figure 7: Drying of bead packing filled with deionized water: water saturation profiles $0,15,21,29,35,39$ and 43 hours after the beginning. 
When the bead packing is filled with microgel suspensions at low initial concentration $\left(c_{0}=3.5 \mathrm{~g} / \mathrm{L}\right.$ ) the overall aspect of the water distribution in time is similar to that for pure water: the water saturation decreases fairly uniformly. However there are two clear differences: the air reaches the bottom of the sample after a slightly longer time, i.e. 18 hours instead of 16 (see Figure 8.a), and a dry front $(h)$ appears and slowly develops after only 10 hours, when the water saturation is still high (i.e. $\phi \approx 0.75$ ). Crucially, this latter effect is at the origin of the decrease of the drying rate observed in that case (see Figure 5): The water vapor must now diffuse through a significant distance before reaching the sample free surface.

These effects are strongly enhanced for higher microgels concentrations: The water profiles are now composed of a dry front $(h)$ rapidly progressing into the sample, while the bottom of the sample water saturation stays close to its initial value (i.e. 1). The air never reaches $z=0 \mathrm{~cm}$ within the duration of our tests (see Figures 8.b,c). Thus the pores are fully filled with fluid in this bottom region. Close to the free surface over an increasing length $(h)$, the pores are completely empty of liquid water; we can measure a dry front from the beginning of measurements (after 2 hours of drying). The size of the saturated bottom region increases with the microgel concentration, so that the intermediate area between this region and the dry region decreases. Extrapolating this observation towards even higher concentration suggests that drying would occur in the form of a straight dry front progressing downwards while the rest of the sample remains saturated.

(a)

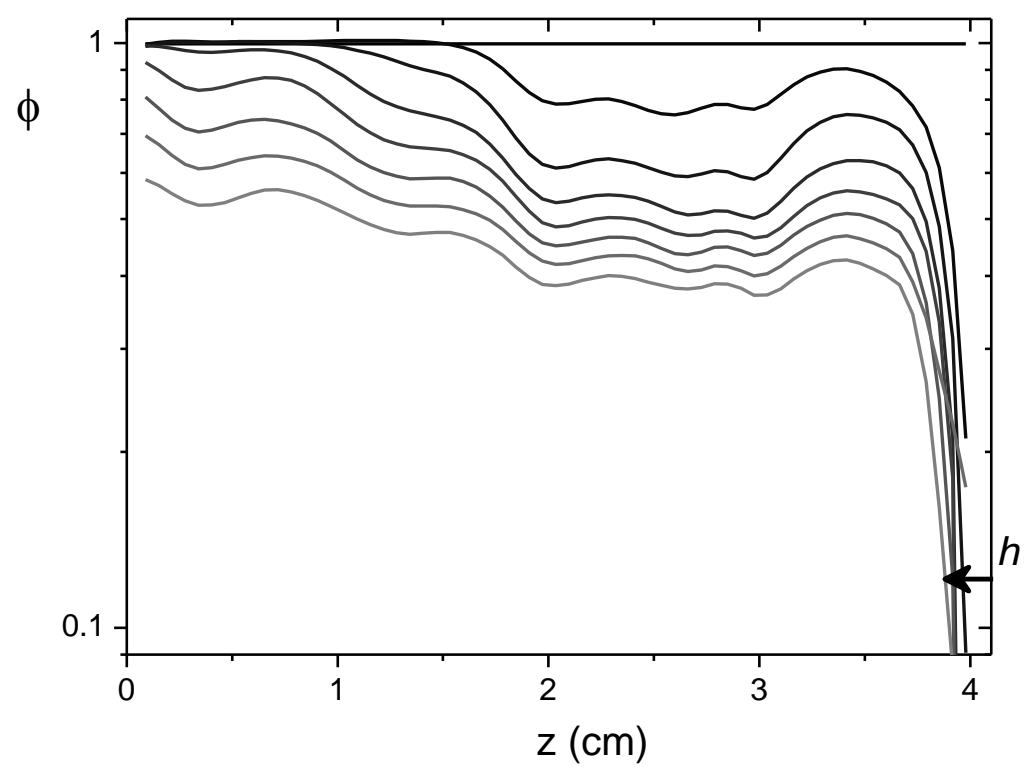


(b)

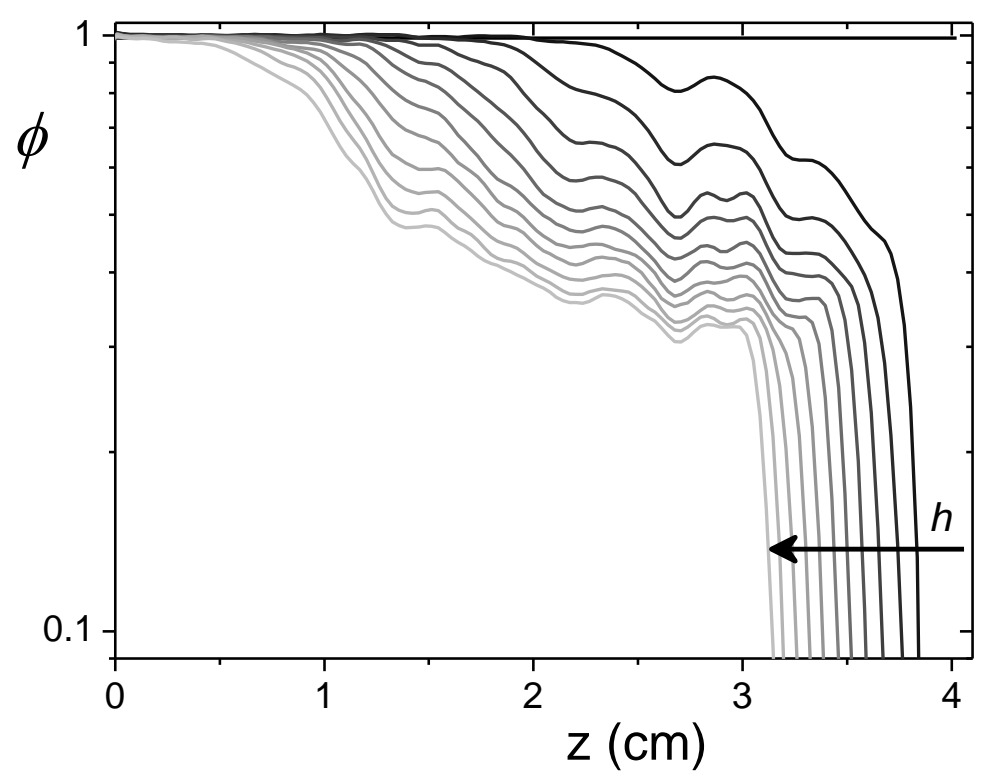

(c)

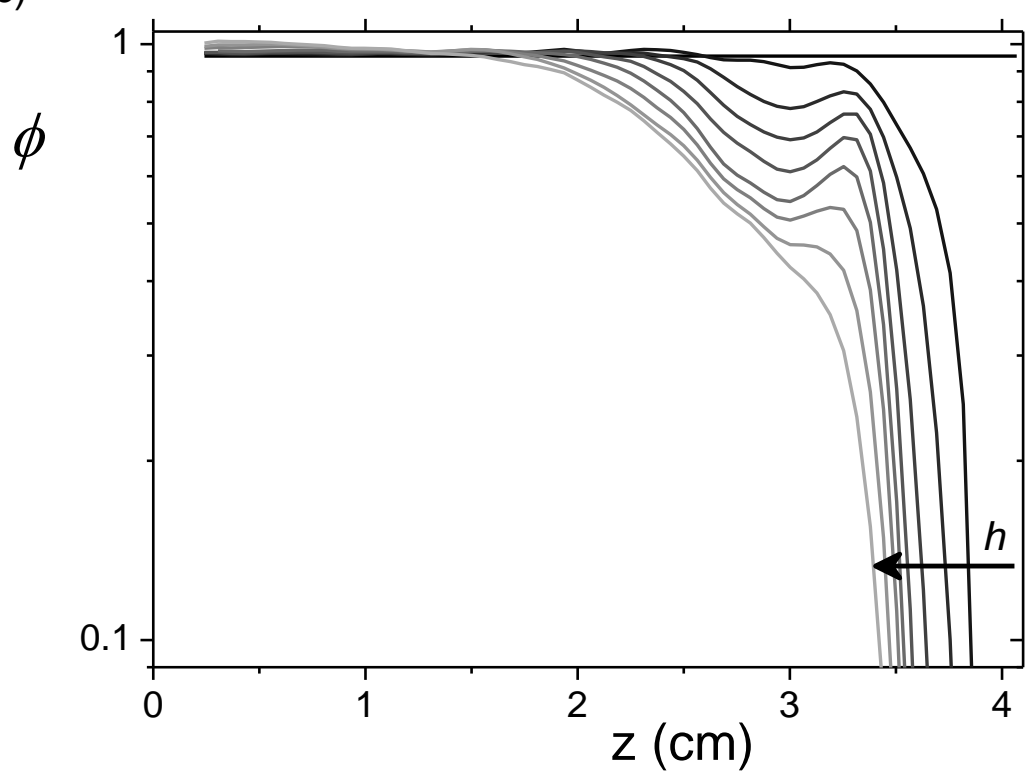

Figure 8: Drying of bead packing filled with microgel suspensions: (a) $c_{0}=3.5 \mathrm{~g} / \mathrm{L}$, (b) $c_{0}=10.4 \mathrm{~g} / \mathrm{L}$, (c) $c_{0}=45.1 \mathrm{~g} / \mathrm{L}$. Water saturation profiles every 10 hours after the beginning. The black line is the initial saturation.

By contrast, the local water distributions observed for silica suspensions as initial interstitial fluids in the sample differ from those observed for microgel suspensions. For low or moderate volume fractions of silica particles (i.e. $\psi_{0}<15 \%$ ), the bulk of the sample has a uniform saturation which decreases homogenously with time. Here we only present such results for $\psi_{0}=10 \%$, keeping in mind that the results in terms of water profiles for other concentrations in that range are qualitatively similar (see Figure 9). Close to the top of the sample the saturation progressively drops to zero, thus forming a 
gradient of saturation. As the sample dries, and thus as the saturation decreases, this gradient region increases in size and advances inside the sample, which results in an apparent dry front slowly progressing inwards. At the end of the experiment, this gradient region extends over a third of the sample for $\psi_{0}=10 \%$. At a given saturation, higher $\psi_{0}$ leads to larger gradient region.

For a higher volume fraction of silica particles, $\psi_{0}=33 \%$, the water profiles significantly differ from those observed at low volume fraction: the water profiles are not uniform. But far from the free surface, water saturation remains close to the initial value up to 56 hours, which means that pores are still fully saturated with liquid, at a time when the sample with pure water is almost dried. Close to the free surface, the sample desaturates partially with a constant residual saturation $\phi \approx 0.2$ (see Figure 10). This residual saturation is twice higher than the one observed for low volume fraction. Its length increases sharply at the end of the experiments, indeed it triples between 40 hours and 80 hours.

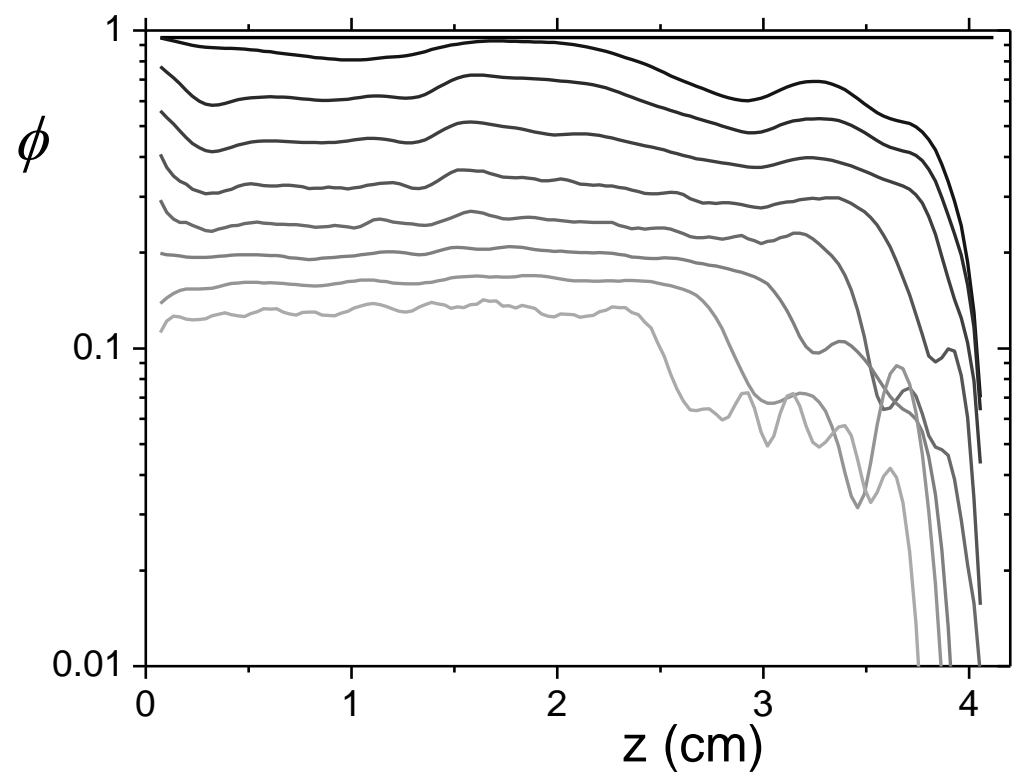

Figure 9: Drying of bead packing filled with silica particle suspensions at $\psi_{0}=10 \%$. Water saturation profiles every 10 hours after the beginning. The black line is the initial saturation. 


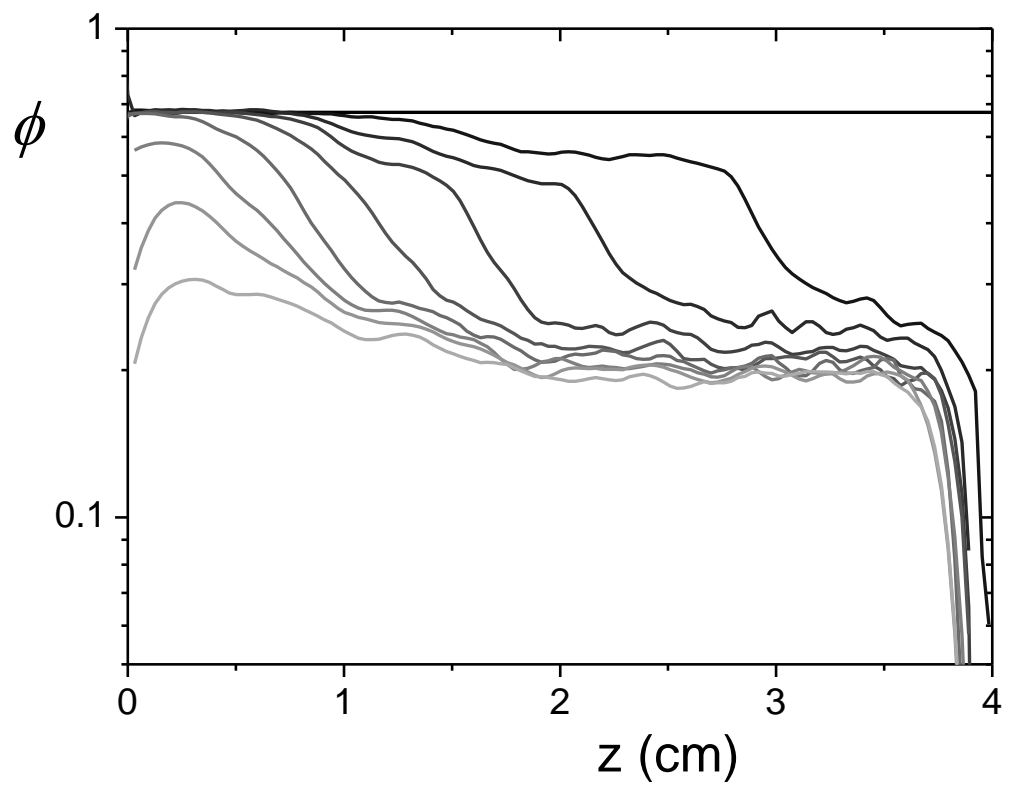

Figure 10: Drying of bead packing filled with silica particle suspensions at $\psi_{0}=33 \%$. Water saturation profiles every 10 hours after the beginning. The black line is the initial saturation.

For these silica suspensions, the particles concentrate in the liquid phase as water evaporates. Thus the particle to liquid ratio $\psi$ increases with time. However, the particle concentration does not remain uniform while drying (see Figure 11). Close to the free surface, the particle concentration is around $60 \%$, corresponding to the maximum random packing fraction of undeformable hard particles, thus the suspension develops a yield stress. In the bottom part of the sample, the particle concentration remains rather low, i.e. between 10 and $20 \%$, so that the suspension is still in a liquid state. The transition between these two regions is relatively abrupt and extends over around $1 \mathrm{~mm}$. Thus by taking the highest value of the derivative of $\psi$, we can determine rather precisely the length $h$ of the region of full compaction. It appears that $h$ increases with time and reaches a third of the sample at the end of the experiment. In fact $h$ corresponds to the transition in water profiles between the low saturation area and the plateau at the bottom (see Figure 9). Similar observations are made on the other three samples with silica particles. 


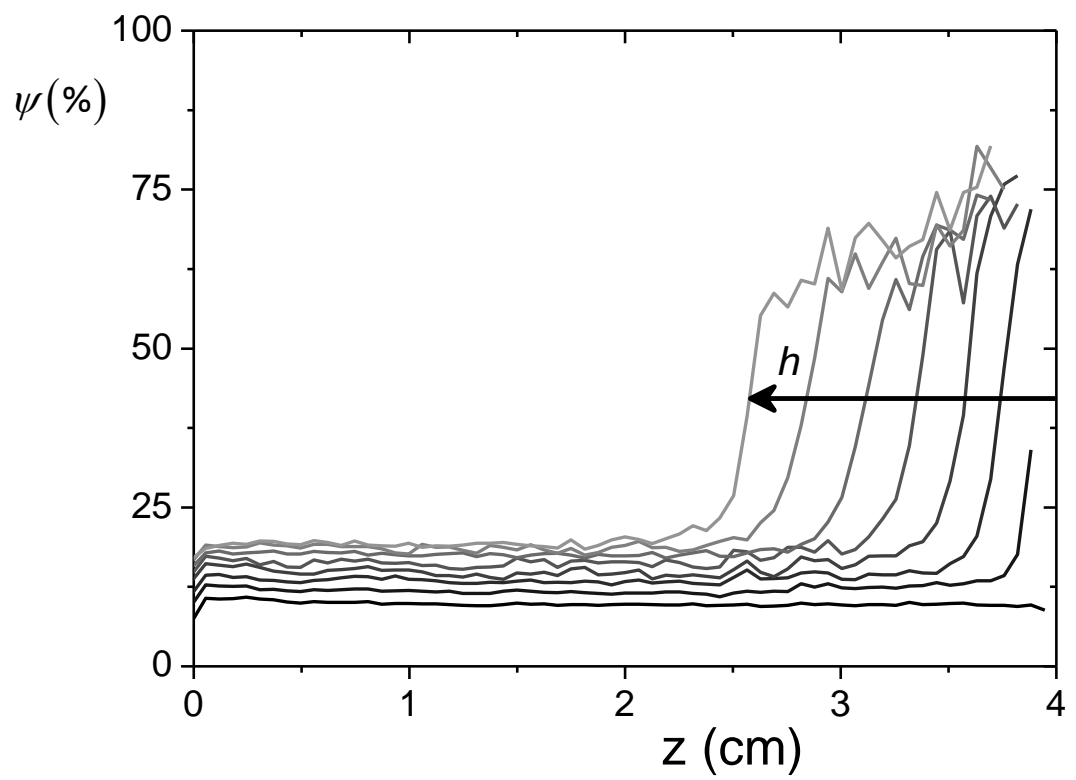

Figure 11: Drying of a bead packing filled with a silica suspension $\left(\psi_{0}=10 \%\right)$. Particle concentration in the liquid as a function of the position in the sample every 10 hours.

To complete our view of this multiphasic system, we must account for particles that do not simply concentrate in the liquid phase but may also move. Figure 12 shows the distribution of particles $\chi_{f}$ in the sample at the end of the experiment. For low volume faction $\left(\psi_{0}=\chi_{0}=10 \%\right)$, we observe that more particles are now present in the first third of the sample close to the surface. Accordingly there is around $70 \%$ of the initial value in the bottom part. Thus particles migrate toward the free surface where they occupy up to $30 \%$ of the pore volume. We observe qualitatively the same migration of particles for $\psi_{0}=5 \%$ as presented in [27]. For high volume fraction ( $\psi_{0}=33 \%$ ) only a slight increase of particles is observed in the first centimeter, and a corresponding small decrease in the bottom part (see Figure 11). The particle distribution remains relatively uniform in the porous structure without noticeable migration toward the free surface. 


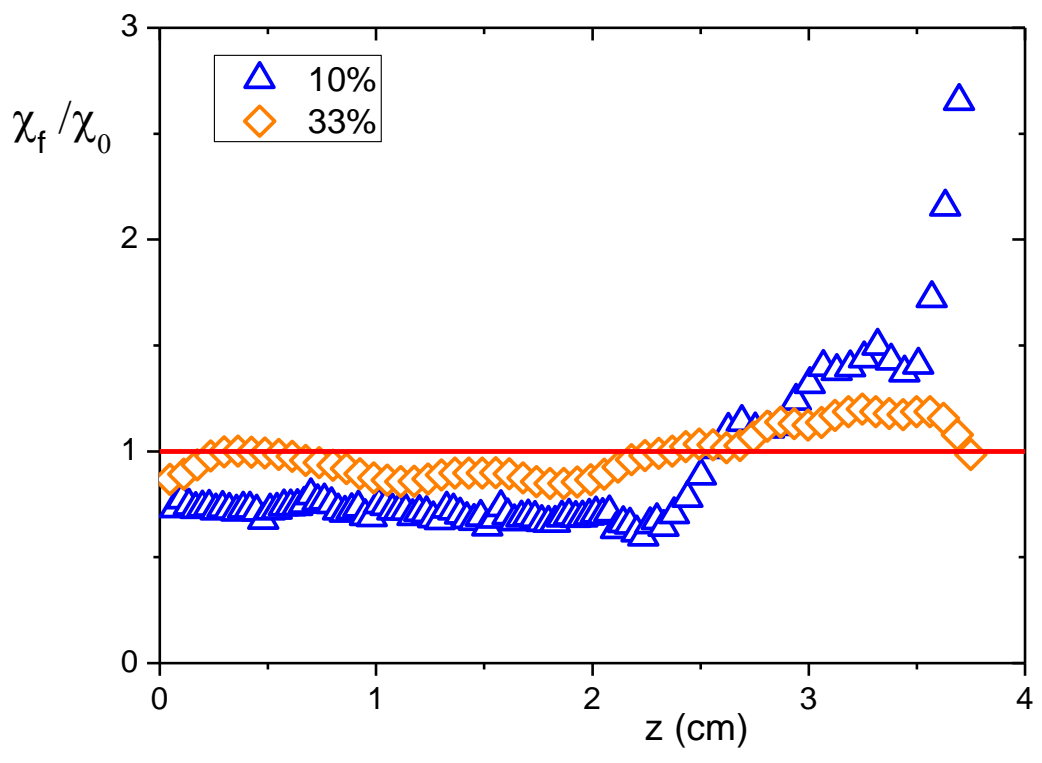

Figure 12: Drying of bead packing filled with silica suspensions: $10 \%$ (blue triangles) and 33\% (orange diamonds). Final particle distribution $\chi_{f}$ normalized by the initial one $\chi_{0}$. The red line is the initial homogeneous state.

\section{Discussion}

Drying of soft particles in a porous medium involves complex concurrent mechanisms. To distinguish the specific impact of soft particles and separate the origin of physical processes, we start by analyzing the case of pure water drying, for which a strong background already exists [12,13], in the light of our MRI data providing some new information on the last stages of drying. Then we present the model based on transport process which describes the drying kinetics of silica (hard) particles in porous material [27], and compare its predictions with our data. Here we specifically discuss the difference on drying properties between (initial) low volume fraction where fluid properties are close to pure water and (initial) high volume fraction for which they significantly differ from pure water, so that the model is unable to predict the experimental trends. This finally yields a framework to understand drying kinetics of soft microgel particles subjected to transport, like hard particles, but which can also themselves deform and dry.

\section{a. Pure water}

Around $75 \%$ of water evaporates at constant drying rate (see Figure 5) corresponding to a uniform water distribution in the sample (see Figure 7), these observations are in good agreement with prediction from Invasion Percolation theory based on capillary effect [42]. In this stage, air inside the sample is saturated by water vapor preventing any evaporation, thus drying occurs only at the free surface. As there is always liquid water at the free surface, this implies that water flows inside the porous medium. Drying at the free surface with constant external conditions insures constant drying rate. From the measured drying rate we can calculate a diffusion length of water vapor above the sample, $\delta^{*}$, considering that water vapor diffuses along one direction according to Fick's law and that 
the air flux is dry, which sets the external vapor density at $n_{0}=0 \mathrm{~g} / \mathrm{m}^{3}$. At the free surface, air is saturated with water vapor, i.e. $n_{\text {sat }}=23.4 \mathrm{~g} / \mathrm{m}^{3}$ (at $25^{\circ} \mathrm{C}$ ). Thus we find the average velocity of liquid water at the free surface $[12,13,43]$ :

$$
V_{e}=\frac{-1}{\phi \rho_{0} S} \frac{\mathrm{d} m}{\mathrm{~d} t}=D \frac{n_{\text {sat }}-n_{0}}{\phi \rho_{0} \delta^{*}}
$$

where $S=9.6 \cdot 10^{-4} \mathrm{~m}^{2}$ is the sample surface area, $\rho_{0}=1000 \mathrm{~kg} / \mathrm{m}^{3}$ the water density and $D=2.7 \cdot 10^{-5} \mathrm{~m}^{2} / \mathrm{s}$ the water vapor diffusion coefficient in the air. As $V_{e} \approx 9.5 \cdot 10^{-8} \mathrm{~m} / \mathrm{s}$, we deduce $\delta^{*}=6.6 \mathrm{~mm}$. This diffusion length can be converted into a distance inside the porous structure by taking into account the effective diffusion length through a porous medium $D_{\text {eff }}=\omega D$ thus $\delta=\omega \delta^{*}=2.25 \mathrm{~mm}$, with $\omega=0.34$ being the porosity. Note that we do not take into account the tortuosity factor besides porosity in this diffusion coefficient, but this does not change at all the analyses of this work.

The capacity of the porous media to provide water to the free surface depends on the capillary pumping $[12,13,14]$. In this experiment, $\frac{\rho_{0} g H}{2 \gamma / r} \approx 0.1(\gamma=70 \mathrm{mN} / \mathrm{m}$ the surface tension), the gravitation effect is small compared to capillary pressure, therefore, the water flows in the porous medium according to Darcy's law with a pressure gradient dominated by the capillary pressure. Thus the maximal velocity is $[12,43,13]$ :

$$
V_{\text {cap }}=\frac{2 G(\phi) F(\omega) \gamma r}{\eta H}
$$

$F$ can be estimated by the Kozeny-Carman formula $F(\omega)=\frac{\omega^{3}}{45(1-\omega)^{2}}=2 \cdot 10^{-3}[44,45], G$ is the decrease of liquid permeability with saturation; in that aim we use the empirical formula [46]: $G(\phi)=\frac{\left(\phi-\phi_{c}\right)^{3}}{\left(1-\phi_{c}\right)^{3}}$ and we assume that the liquid water network disconnects at $\phi_{c}=0.05$. From these values, we obtain the initial velocity due to such capillary flow $V_{\text {cap }}(\phi=1) \approx 2.7 \cdot 10^{-4} \mathrm{~m} / \mathrm{s}$. Thus $V_{c a p}(\phi=1) \gg V_{e}(\phi=1)$ and capillary pumping is fast enough to provide liquid water to compensate evaporation at the free surface, which explains that in this regime the saturation remains homogeneous.

From the MRI data, we can establish the critical saturation corresponding to the end of this regime. From $\phi \approx 0.2$, we observe a dry area progressing in the sample (see Figure 7 ). In fact, for saturation lower than $\phi \approx 0.2$ we have

$$
V_{\text {cap }}(\phi=0.2)=10^{-6} \mathrm{~m} / \mathrm{s} \approx V_{e}(\phi=0.2)=5 \cdot 10^{-7} \mathrm{~m} / \mathrm{s}
$$

which implies that $V_{e}$ and $V_{\text {cap }}$ are of the same order of magnitude; based on equations (4) and (5) the equality is reached for $\phi=0.17$. Therefore, water evaporation at the sample free surface is too demanding and evaporative water is no longer replaced, and a dry front progresses inside the porous medium. Then water vapor above the sample must diffuse over an increasing length, which slows down the drying rate. For $\phi<0.2$, with the help of MRI we can measure the position of the dry front 
$h$. The diffusion length is now $\delta+h$ and according to equation (5), the drying rate $\frac{\mathrm{d} m}{\mathrm{~d} t}$ should decrease by a factor $\delta /(\delta+h)$. Our data (see Figure 13) are in excellent agreement with this mechanism, we indeed find $\frac{\mathrm{d} m}{\mathrm{~d} t} / \frac{\mathrm{d} m}{\mathrm{~d} t} \approx \delta /(\delta+h)$.

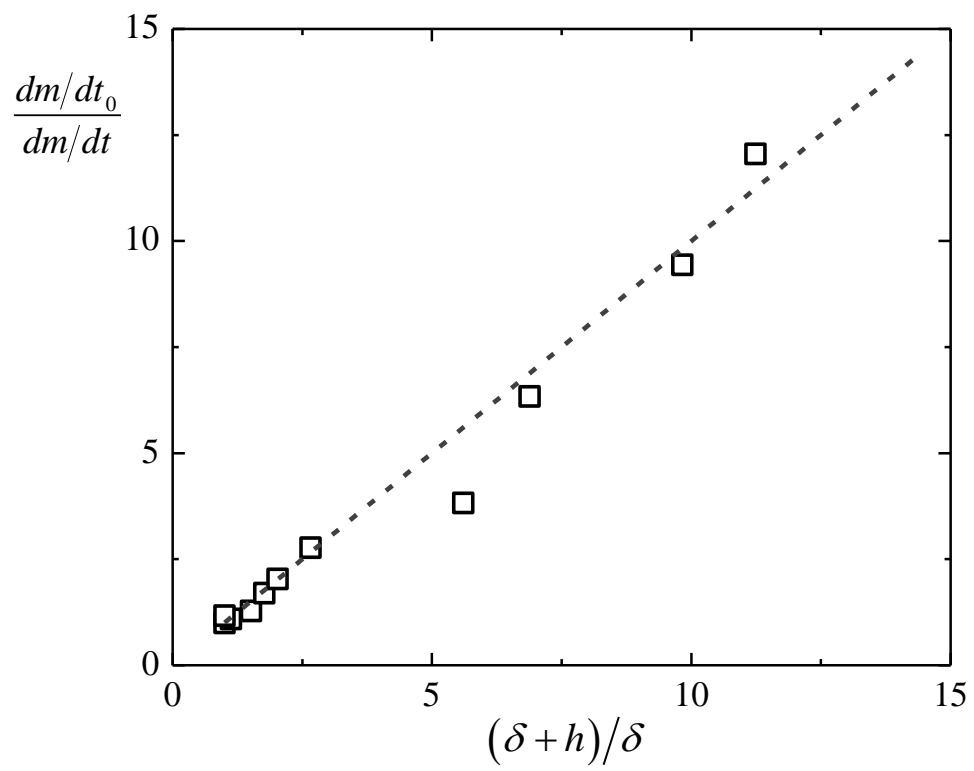

Figure 13: Bead packing filled with pure water: inverse of the drying rate normalized by the initial value as a function of the dry front thickness. The dash line is $y=x$.

\section{b. Silica particles}

Let us first recall the principles of the model presented in [27] for describing the transport and drying processes of low concentration $\left(\psi_{0} \leq 15 \%\right)$ silica suspensions in bead packing. In this case, the saturation profiles have a plateau similar to the case of pure water (see Figure 9). Thus the same physical process is likely at work and capillary pressure flows water towards the free surface. In fact the capillary velocity $V_{\text {cap }}$ in equation (6) depends on the fluid via the ratio of surface tension to viscosity $\gamma / \eta$. While, $\gamma$ decreases slightly upon silica particle addition, it maintains the same order of magnitude, and $\eta$ increases by less than a factor 10 for $\psi_{0}<20 \%$ ( see Figure 2). Thus the suspension characteristics are close to those of water. Initially, $V_{\text {cap }} \approx 10^{-5} \mathrm{~m} / \mathrm{s} \gg V_{e}$, so that capillary flow is dominant for high saturation $\phi$. The liquid flows towards the top of the sample and a priori tends to transport the suspended particles which accumulate at the top liquid-air interface before the sample free surface, at a maximum volume fraction $\psi_{m} \approx 60 \%$. Nevertheless the particle concentration may also tend to equilibrate due to the diffusion of these colloidal particles. The characteristics diffusion velocity over the sample can be estimated by Stokes-Einstein diffusion over the length of the sample as: 


$$
V_{d}=\frac{k_{B} T}{3 \pi \eta d H}
$$

where $k_{B}$ is the Boltzmann's constant. We find $V_{d} \approx 3 \cdot 10^{-9} \mathrm{~m} / \mathrm{s} \ll V_{e}$, which implies that any redispersion effect due to diffusion is negligible compared to capillary driven flow. The particles accumulate in the liquid films in the pores the closest to the free surface and the compacted front progresses, i.e. the position of the liquid-air interface the closest to the sample top recedes. Such a process effectively predicts our observations concerning the evolution of the water profiles in time (see Figure 9) and the distribution of particle concentration (see Figure 11).

In fact this compacted region limits the drying rate. Indeed, it forms a new porous structure below the sample free surface with $r_{\text {nano }} \approx 3 \mathrm{~nm}$. Here, we may applied the same arguments on capillary flow for pore $10^{4}$ smaller, which has a strong impact on $V_{\text {cap }}$ which scales as $r$ (see equation (6)). Thus capillary flow through the compacted front can sustain water evaporation at $V_{e}$ only if it has a size $h<1 \mathrm{~mm}$. For larger size, the possible liquid transport through it is much slower than the water vapor diffusion in the air of the main porous network. Additionally, the water contained in the small pores between packed particles plays a negligible role on the evaporation process because the corresponding water mass is very small compared to the remaining water. We could expect that this compacted region becomes partly dry at some time but this is not supported by the measurements. This might be due to the Kelvin effect, allowing the liquid water to be at equilibrium with unsaturated air in nanometric pores $[47,48]$. Thus the compacted area acts as a dry front disconnecting the main liquid films from the free surface. Note that as the initial volume fraction of particles is higher the size of the compacted front increases faster and the drying rate decreases faster.

Particle concentrations reach $60 \%$ in the liquid films (see Figure 11) but these films occupy a decreasing fraction of the pore volume as water evaporates. Thus the main part of the pore structure close to the free surface is not clogged with particles (see Figure 12). Therefore particle deposit close to the surface does not block water vapor diffusion. As for pure water, we find that the drying rate decreases with the compaction front $h: \frac{\mathrm{d} m}{\mathrm{~d} t} / \frac{\mathrm{d} m}{\mathrm{~d} t}{ }_{0} \approx \delta /(\delta+a \cdot h)$ with $a=1.5$ (see Figure 14). This validates our assumption of diffusion of water vapor over the length of the compacted area $h$. However the proportionality factor $a$ is higher than for pure water. This may be due to an increase of the effective diffusion length due to a higher tortuosity as a result of particle deposits in the sample. A striking feature however is that $a$ is a constant independent of the initial particle volume fraction. For a given length, the presence of a small amount of particles is enough to reduce the water vapor diffusion 1.5 times more than in the absence of particles. Due to capillary effect, deposit of particle is at the contact points between glass beads. Our interpretation is that these points are critical to diffusion; even without clogging the pore space, particle deposit can reduce significantly diffusion. An alternative interpretation is an evolution of surface diffusion length $\delta$ in the very first times of the drying experiment, possibly due to particle accumulation close to the surface. Note that we compute $\delta$ based on equation (5) for each experiments. Indeed dividing $\delta$ by $a$ would also explain the $\mathbf{1 . 5}$ slope observed in Fig. 14. Again, it is striking that this corrective factor does not depend on initial particle concentration. To this regard, slope 1 found for pure water in fig. 13 could also be regarded as fortuitous. Further work would certainly be necessary to fully understand this effect. Both interpretations lead to quantitatively equivalent descriptions. Without any loss of generality, the tortuosity hypothesis will then be used in the following. 
Under these conditions the drying rate $J=\frac{-1}{\omega S \rho_{0}} \frac{\mathrm{d} m}{\mathrm{~d} t}$ may be written as

$$
J=\frac{D n_{\text {sat }}}{\rho_{0}(\delta+a h)}
$$

The decrease of the drying rate is coupled with the progression of the compacted front. The drying rate is the driving mechanism of particle accumulation. As particles pack, the capillary flow still occurs in the bottom part of the sample, where the saturation is $\phi$, and the liquid films, occupying a fraction $\phi /\left(1-\psi_{0}\right)$ of the pore volume, continuously provide water and particles to the compacted front. A small advance of the compacted front, $d h$, is associated with a decrease of the saturation $\mathrm{d} \phi$ and the local concentration increases from $\psi_{0}$ to $\psi_{m}$. Moreover the bottom part of the sample has a uniform saturation over a length, $H-h$. Thus, the particle volume is kept, the particles arriving in the packed area correspond to the particles removed from the bottom part of the sample:

$$
\left(\psi_{m}-\psi_{0}\right) \phi \frac{\mathrm{d} h}{\mathrm{~d} t}=-\psi_{0}(H-h) \frac{\mathrm{d} \phi}{\mathrm{d} t}
$$

The water mass conservation completes the description of the process. In addition, to the advected water, the water amount between $h$ and $h+d h$ decreases to leave space to the advected particles, the water fraction thus turns from $1-\psi_{0}$ to $1-\psi_{m}$, so that the water loss is $\left(\psi_{m}-\psi_{0}\right)$. Finally, the evaporated water has two origins, the water flow from lower regions of the sample and from the progression of the compacted area:

$$
J=\left[\left(\psi_{m}-\psi_{0}\right)+\left(\psi_{m}-\psi_{0}\right) \frac{1-\psi_{0}}{\psi_{0}}\right] \frac{\phi}{1-\psi_{0}} \frac{\mathrm{d} h}{\mathrm{dt}}
$$

Using equations $(8,9,10)$, we solve numerically the coupled differential equations and predict the drying kinetics, which appears to be in very good agreement with experiments for $\psi_{0}=5-10-15 \%$ (see Figure 6.a ). A higher particle volume fraction leads to a larger packed area and thus to a slower drying kinetics. Thus we have a model based on a few assumptions (describing water vapor diffusion, particle and water conservations) and measurable parameters: initial drying rate, porosity, maximum packing fraction, effective diffusion in the porous structure, which depict very well the drying kinetics and the final particle distribution [27].

However, the model does not represent the drying kinetics for $\psi_{0}=33 \%$ (see Figure 6 ), the sample dries much faster than predicted. Actually we observe that the particles do not migrate toward the free surface of the sample (see Figure 12), and the water profiles are not uniform in the bottom part, the pores remain at the initial saturation (see Figure 10). This means that the fluid does not flow as expected as a result of capillary effects. This is certainly due to the fact that at such a high initial concentration, the suspension viscosity is much higher than for pure water or low volume fraction of silica particles: $\eta_{r} \approx 500$ (see Figure 2). Thus $V_{\text {cap }} \approx 5 \cdot 10^{-7} \mathrm{~m} / \mathrm{s}$ is of the same order of magnitude as $V_{e}$ $\left(\approx 10^{-6}-10^{-7} \mathrm{~m} / \mathrm{s}\right)$ and the capillary flow is not fast enough to provide water to the free surface in the form of a bulk flow, i.e. water and particles. Nevertheless water remains close to the free surface until 
the end of the experiment, suggesting that the water flows between the particles towards the free surface.

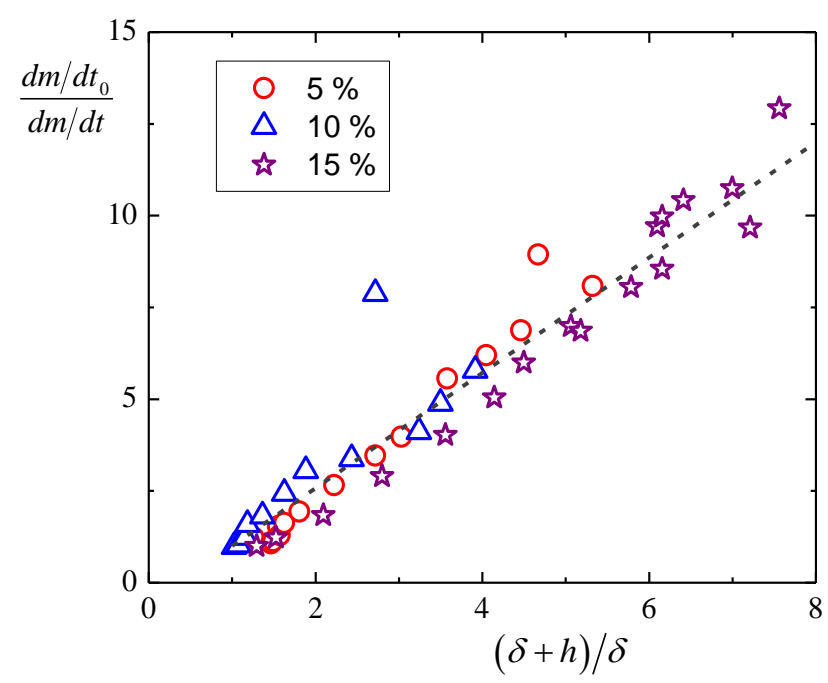

Figure 14: Bead packing filled with silica suspensions (same symbols as in Figure 5): inverse of the drying rate normalized by the initial value as a function of the dry front thickness. The slope of the dash line is 1.5 .

\section{c. Microgel suspensions:}

In this section we will use our understanding of drying and advective transport of both pure water and particles to consider deformable particle suspension in a granular packing. Microgels particles are mainly made of water [49], but the water inside particles does not contribute to the flow of the suspension as the mesh size of the polymer network is nanometric [50]. Considering water inside a microgel particle as part of this particle, we deduce an equivalent volume fraction of particles for microgel suspensions similarly to silica particle suspensions. Under these conditions the transport properties through the porous medium should be similar as long as the microgel particles keep the same shape and size, and the drying characteristics should be the same. The observed differences necessarily find their origin in the particle properties, i.e. the compressibility of microgel particles and the ability to expel water.

For $c_{0}=3.5 \mathrm{~g} / \mathrm{L}$, the drying kinetics is slower than for pure water; the drying rate decreases by a factor 2 from $\phi \approx 0.9$ to $\phi \approx 0.4$. Nevertheless, similarly to pure water, the water distribution is fairly uniform in the main part of the sample. In addition a dry front appears below the free surface and grows: at the end of the experiment it has reached a length $h \approx 1.5 \mathrm{~mm}$, along which water vapor must diffuse. Under these conditions and using the same tortuosity $a$ than for silica particle experiments, $\delta /(\delta+a \cdot h) \approx 0.5$, and according to equation (9), the drying rate should decrease by $50 \%$ over the test duration, which is in good agreement with the observation (see Figure 5). This analysis is further confirmed by the fact that, as for silica suspensions, equation (9) appears to be valid all along the drying process for dilute microgel suspension (see red circles on Figure 15). We conclude that in this case the decrease of the drying rate is mainly associated with a vapor diffusion over the dry region. 
The physical origin of the dry region is analogous to that for the compacted region for silica particle suspensions. Microgel particles, which have a much larger size (i.e. $1 \mu \mathrm{m}$ ) than silica particles are advected by the liquid flow which is still controlled by capillarity and particle diffusion is negligible. Thus the transport and accumulation process during drying is similar to that for silica suspensions, and the dry region should correspond to a compacted region of microgel particles. However the exact characteristics of this region, and in particular the characteristics of the microgel compaction are unknown. We consider that microgel particles, as silica particles, migrate towards the free surface and accumulate at a maximum packing concentration $\psi_{m}=1$. Therefore we define the microgel particle volume fraction as $\psi_{0}=c_{0} / c_{m}$, using the similarity of the viscosity as a function of the concentration curves for silica and microgel suspensions, at low concentration (see Figure 2). As we measure an area close to the free surface completely dry, we conclude that microgels release their water faster than the drying kinetics. Flow inside compressed microgels is possible but the permeability is very low in the nanometric mesh of the polymer network. Similarly to silica particles packing area, flow appears to be too slow to provide water fast enough to counter evaporation. Finally the microgel particles shrink and loose water to finally reach a dry state at $c_{d}=1000 \mathrm{~g} / \mathrm{L}$, considering microgel density to be 1. Thus they release an extra fraction of water: $\left(1-c_{m} / c_{d}\right)$. The mechanism can be modelled on the basis of the same physical processes as for silica particles with two differences: (i) the value of the maximum packing fraction and (ii) the possibility of water evaporation from the interior of the particles. Equations (9) and (10) are unchanged as the relation between the dry front and the decrease of the drying rate is the same as for silica particles, and particle migration should not differ. Equation (11) is modified by adding a third term taking into account the extraction of water from the microgels:

$$
J=\left[\left(1-\psi_{0}\right)+\frac{\left(1-\psi_{0}\right)^{2}}{\psi_{0}}+\left(1-\frac{c_{m}}{c_{d}}\right)\right] \frac{\phi}{1-\psi_{0}} \frac{\mathrm{d} h}{\mathrm{~d} t}
$$

We can again solve numerically equations $(8,9,11)$ and predict the drying kinetics (see Figure 5) without any fitting parameter. The initial decrease is not described by the model but the subsequent drying kinetics is in very good agreement with the migration-diffusion model.

For higher concentration of microgels $\left(c_{0}=10.4-45.1 \mathrm{~g} / \mathrm{L}\right)$, the fully saturated area at the bottom of the sample suggests that capillarity is not sufficient to flow water out of the saturated area toward the free surface. Indeed the fluid properties are different from pure water, modifying its capacity to flow under capillary pressure. In equation (6), the capillary flow $\left(\mathrm{V}_{\text {cap }}\right)$ depends on the fluid properties through $\gamma / \eta_{r}$. The surface tension $\gamma$ for microgels suspensions is about half the one of pure water [51]. Moreover the viscosity increases even more significantly by a factor 7 to 35 , and is the main factor in reducing $\mathrm{V}_{c a p}$. The resulting $\mathrm{V}_{c a p}$ decreases by a factor 14 to $70: \mathrm{V}_{c a p} \approx 2 \cdot 10^{-5}-4 \cdot 10^{-6} \mathrm{~m} / \mathrm{s}$. Thus capillary flow is still higher than the initial flow induced by drying $V_{e} \approx 10^{-7}-10^{-6} \mathrm{~m} / \mathrm{s}$ but it appears to be not sufficient to ensure uniform water distribution (see Figure $8 . b$ and c). In complete absence of water flow the sample should be divided into two parts: a top dry area, and a fully saturated bulk. In addition to these two areas we observed an intermediate area where water saturation decreases, suggesting that water equilibrates over a limited length. In fact $\mathrm{V}_{\text {cap }}$ is proportional to $1 / H$, by considering a smaller length than the height of the sample, the capillary flow is fast enough to compensate drying and equilibrate water distribution. Nevertheless, as the evaporated water around the free surface is not replaced, the dry area increases. Once again equation (9) is verified at any time 
during the drying of the two samples (see Figure 15): the dry area (of thickness $h$ ) induces an increase of the diffusion length of water vapor. Since here water is essentially present below the dry area, we may write:

$$
m=S \omega \rho_{0}(H-h)
$$

From equations (9) and (14) we deduce that the mass loss evolves as $m_{0}-m \propto \sqrt{t}$ [43] which is consistent with the observation of a slope $1 / 2$ in the logarithmic scale of Figure 5 . For high concentration, the release of water by microgels does not appears as a limiting factor of the drying kinetics.

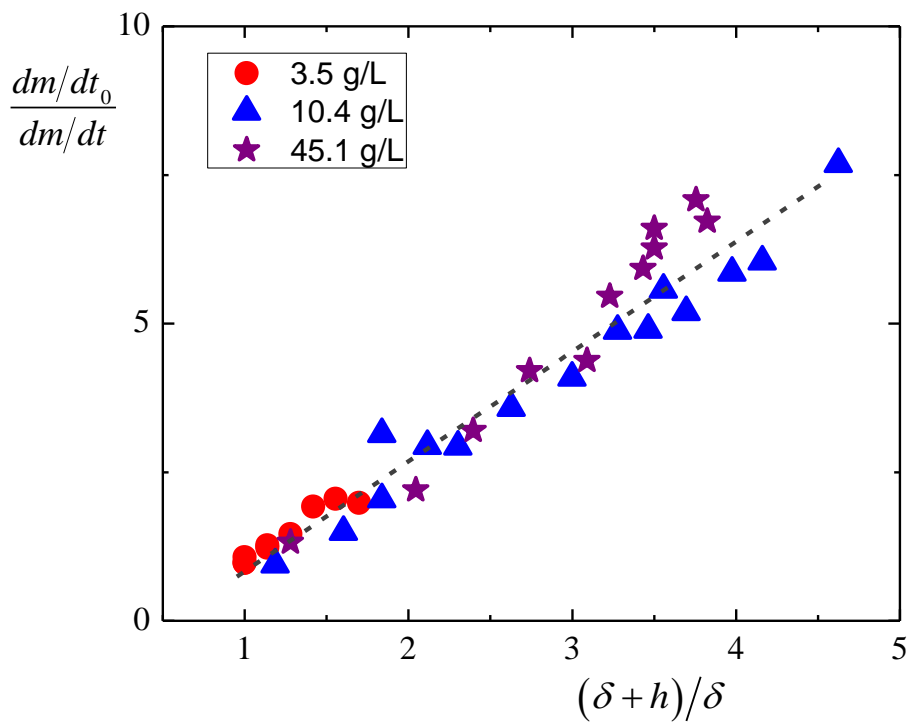

Figure 15 : Bead packing filled with microgel suspensions (same symbols as in Figure 4): inverse of the drying rate normalized by the initial value as a function of the dry front thickness. The slope of the dash line is 1.5 .

\section{Conclusions}

MRI is a powerful tool to quantify water distribution in porous structures. Pure water represents the fastest drying kinetics of a porous medium. Capillary pumping plays a major role providing the surface with water from the inside and equilibrating the water uniformly. In this simple situation, our results are compatible with the hypothesis under which the vapor diffusion length stays constant at the initial value set by external conditions.

Whereas the drying rate remains constant during a long period for pure water due to capillary flow providing liquid water to the surface, this is in contrast with a microgel suspension made of soft polymeric particles where the drying rate decreases immediately and significantly. From measurements of the spatial water saturation with MRI, we identify different situations with or without water flow. In the light of experiments with suspensions of solid particles, we model the drying kinetics in low viscous fluid. In this case, as capillarity dominates, particles are advected toward the free surface where they pack. Packed microgels compress and leave a film of dry polymers. Microgels release their water faster than the drying kinetics, but the structure of microgels may be an important parameter. Remarkably, a simple model assuming particle transport and accumulation at the first air-liquid interface below the sample free surface and a modification of sample tortuosity in 
the presence of particles well describes our observations without fitting parameters. The high viscosity of the microgel suspension of increasing concentration inhibits flow towards the free surface which induces a recession of the air-liquid interface and a dry front appears.

The drying process with microgel suspensions thus significantly differs from that with solid colloidal suspensions due to capillary pumping which reequilibrates the saturation throughout the medium depending on the fluid composition via the ratio $\gamma / \eta$. As it decreases so does the capacity of the porous medium to sustain a high demand of evaporation from the free surface. For a sufficiently high viscosity, the water does not flow; accordingly the pores stay filled at the bottom of the sample. Over a limited length, capillarity does provide water flow but is insufficient; the pores which are close to the surface tend to dry.

Thus disconnecting the water network or moving away the top air-liquid interface from the sample free surface induces vapor diffusion over larger distances. This interpretation is a unique method of decreasing the drying rate in various porous media samples containing dispersed or dissolved materials. By knowing the initial drying conditions and composition of our porous medium we can predict with the suitable physical mechanisms the drying kinetics and particle migration. This gives us a tool to tune the drying kinetics as we can control the diffusion length by changing the fluid properties, i.e. viscosity, particles softness and compaction. This approach provides physical parameters to reduce drying time or increase water retention in porous media. 


\section{References}

[1] A. S. Mujumdar, "Handbook of Industrial Drying," CRC Press, London, 2006.

[2] M. S. Johnson, "The Effects of Gel-forming Polyacrylamides on Moisture Storage in Sandy Soils," J. Sci. Food Agric., vol. 35, pp. 1196-1200, 1984.

[3] K. Kazanski and S. Dubrovski, "Chemistry and Physics of agricultural hydrogels," Advances in polymer Sc., vol. 104, pp. 97-133, 1992.

[4] M. R. Islam, A. E. Eneji, C. Ren, J. Li and Y. Hu, "Impact of water-saving superabsorbent polymer on oat (Avena spp.) yield and quality in an arid sandy soil," Scientific Research and Essays, vol. 6, no. 4, pp. 720-728, 2011.

[5] A. K. Bhardwaj, I. Shainberg, D. Goldstein, D. N. Warrington and G. J. Levy, "Water Retention and Hydraulic Conductivity of Cross-Linked Polyacrylamides in Sandy Soils," Soil Sc. Society of America Jour., vol. 71, no. 2, pp. 406-412, 2006.

[6] R. A. Herrett and P. A. King, "Plant growth medium," US Patent, p. US 3336129 A, 1967.

[7] C. Brumaud, H. Bessaies-Bey, C. Mohler, R. Baumann, M. Schmitz, M. Radler and N. Roussel, "Cellulose etheres and water retention," Cem. Concr. Res., vol. 53, pp. 176-184, 2013.

[8] C. Marlière, E. Mabrouk, M. Lamblet and P. Coussot, "How water retention in porous media with cellulose ethers works," Cem. Concr. Res., vol. 42, pp. 1501-1512, 2012.

[9] M. Wyrzykowski, P. Trtik, B. Münch, J. Weiss, P. Vontobel and P. Lura, "Plastic shrinkage of mortars with shrinkage reducing admixture and lightweight aggregates studied by neutron tomography," Cement and Concrete Research, vol. 73, pp. 238-245, 2015.

[10] Y. Wei and D. J. Durian, "Rain water transport and storage in a model sandy soil with hydrogel particle additives," Eur. Phys. J. E, vol. 37, p. 97, 2014.

[11] E. Verneuil and D. J. Durian, "Permeability of mixed soft and hard granular material: hydrogels as drainage modifiers.," The European Physical Journal E, vol. 34, no. 65, 2011.

[12] P. Coussot, "Scaling approach of the convective drying of a porous medium," Eur. Phys. J. B, pp. 15, 557-566, 2000.

[13] A. Yiotis, D. Salin, E. Tajer and Y. Yortsos, "Drying in porous media with gravity-stabilized fronts: Experimental results," Physical Review E, vol. 86, p. 026310, 2012.

[14] F. Chauvet, P. Duru, S. Geoffroy and M. Prat, "Three Periods of Drying of a Single Square Capillary Tube," Physical Review Letters, vol. 103, no. 12, pp. 1-4, 2009.

[15] N. Shokri and D. Or, "What determines drying rates at the onset of diffusion controlled stage-2 evaporation from porous media?," Water Resources Research, vol. 47, no. 9, pp. 1-8, 2011.

[16] J. Van Brakel, "Mass transfer in convective drying," Adv. Drying, vol. 1, pp. 217-267, 1980. 
[17] P. Lehmann, S. Assouline and D. Or, "Characteristic lengths affecting evaporative drying of porous media," Phys. Rev. E, vol. 77, p. 056309, 2008.

[18] L. Pel, A. Sawdy and V. Voronina, "Physical principles and efficiency of salt extraction by poulticing," Journal of Cultural Heritage, vol. 11, no. 1, p. 59-67, 2010.

[19] C. Shen, V. Lazouskaya, Y. Jin, B. Li, Z. Ma, W. Zheng and Y. Huang, "Coupled factors influencing detachment of nano- and micro-sized particles from primary minima," Journal of Contaminant Hydrology, Vols. 134-135, pp. 1-11, 2012.

[20] M. D. Seck, M. Van Landeghem, P. Faure, S. Rodts, R. Combes, P. Cavalié, E. Keita and P. Coussot, "The mechanisms of plaster drying," J Mater Sci, vol. 50, pp. 2491-2501, 2015.

[21] H. P. Huinink, L. Pel and M. A. J. Michels, "How ions distribute in a drying porous medium: A simple model," Physics of Fluids, vol. 14, no. 4, pp. 1389-1395, 2002.

[22] V. Voronina, L. Pel and K. Kopinga, "Effect of osmotic pressure on salt extraction by a poultice," Construction and Building Materials, vol. 53, pp. 432-438, 2014.

[23] N. Shahidzadeh-Bonn, J. Desarnaud, F. Bertrand, X. Chateau and D. Bonn, "Damage in porous media due to salt crystallization," Physical Review E, vol. 81, p. 066110, 2010.

[24] S. Gupta, H. P. Huinink, M. Prat, L. Pel and K. Kopinga, "Paradoxical drying of a fired-clay brick due to salt crystallization," Chemical Engineering Science, vol. 109, pp. 204-211, 2014.

[25] J. Desarnaud, H. Derluyn, L. Molari, S. de Miranda, V. Cundde and N. Shahidzadeh, "Drying of salt contaminated porous media: Effect of primary and secondary nucleation," J. of apllied Physics, vol. 118, p. 114901, 2015.

[26] F. De Larrard, Concrete Mixture Proportioning, London: E \& FN Spon, 1999.

[27] E. Keita, P. Faure, S. Rodts and P. Coussot, "MRI evidence for a receding-front effect in drying porous media," Physical Review E, vol. 87, no. 6, p. 062303, 2013.

[28] P. Faure and P. Coussot, "Drying of a model soil," Physical Review E, vol. 82, no. 3, pp. 1-12, 2010.

[29] T. Kajiya, E. Nishitani, T. Yamaue and M. Doi, "Piling-to-buckling transition in the drying process of polymer solution drop on substrate having a large contact angle," Physical Review E, vol. 73, p. 011601, 2006.

[30] J. Mattsson, H. M. Wyss, A. Fernandez-Nieves, K. Miyazaki, Z. Hu, D. R. Reichman and D. A. Weitz, "Soft colloids make strong glasses," Nature, vol. 462, pp. 83-86, 2009.

[31] L. A. Lyon and A. Fernandez-Nieves, "The Polymer / Colloid Duality of Microgel Suspensions," The Polymer/Colloid Duality of Microgel Suspensions, vol. 63, pp. 25-43, 2012.

[32] P. Menut, S. Seiffert, J. Sprakel and D. A. Weitz, "Does size matter? Elasticity of compressed suspensions of colloidal- and granular-scale microgels," Soft Matter, vol. 8, pp. 156-164, 2012. 
[33] E. Keita, "Physique du séchage des sols et des matériaux de construction," PhD Thesis (in french), Paris-Est Uni., 2014.

[34] E. Di Giuseppe, A. Davaille, E. Mittelstaedt and M. François, "Rheological and mechanical properties of silica colloids: from Newtonian liquid to brittle behaviour," Rheol Acta, vol. 51, pp. 451-465, 2012.

[35] J. B. Madeline, M. Meireles, C. Bourgerette, R. Botet, R. Schweins and B. Cabane, "Restructuring of Colloidal Cakes during Dewatering," Langmuir, vol. 23, no. 4, pp. 1645-1658, 2007.

[36] H. Y. Carr and E. M. Purcell, "Effects of Diffusion on Free Precession in Nuclear Magnetic Resonance Experiments," Phys. Rev., vol. 94, p. 630, 1954.

[37] S. Meiboom and D. Gill, "Modified Spin-Echo Method for Measuring Nuclear Relaxation Times," Rev. Sci. Instrum. , vol. 29, p. 688, 1958.

[38] S. Rodts, J. Boujlel, B. Rabideau, G. Ovarlez, N. Roussel, P. Moucheront, C. Lanos, F. Bertrand and P. Coussot, "Solid-liquid transition and rejuvenation similarities in complex flows of thixotropic materials studied by NMR and MRI," Phys. Rev. E, vol. 81, p. 021402, 2010.

[39] S. V. Dvinskikh, K. Szutkowski and I. Furo, "MRI profiles over very wide concentration ranges: Application to swelling of a bentonite clay," Journal of Magnetic Resonance, vol. 198, no. 2, pp. 146-150, 2009.

[40] P. Faure and S. Rodts, "Proton NMR relaxation as a probe for setting cement pastes," Magnetic Resonance Imaging, vol. 26, no. 8, pp. 1183-1196, 2008.

[41] J.-P. Korb, "NMR and nuclear spin relaxation of cement and concrete materials," Current Opinion in Colloid \& Interface Science, vol. 14, no. 3, pp. 192-202, 2009.

[42] A. G. Yiotis, I. N. Tsimpanogiannis, A. K. Stubos and Y. C. Yortsos, "Pore-network study of the characteristic periods in the drying of porous materials," J. of Colloid and Interface Sc., vol. 297, p. 738-748, 2006.

[43] B. Camassel, N. Sghaier, M. Prat and S. Ben Nasrallah, "Evaporation in a capillary tube of square cross-section: application to ion transport," Chemical Engineering Science, vol. 60, pp. 815-826, 2005.

[44] J. Kozeny, "Ueber kapillare Leitung des Wassers im Boden," Stizungsber Akad Wiss Wien, vol. 136, pp. 271-306, 1927.

[45] P. C. Carman, "Permeability of saturated sands, soils and clays," J Agric Sci, vol. 29, p. 263, 1939.

[46] I. Chatzis and F. A. Dullien, "Modelling pore structure by $2 \mathrm{~d}$ and $3 \mathrm{~d}$ networks with application to sandstones," Journal of canadian Petroleum Technology, vol. 16, pp. 97-108, 1977.

[47] O. Coussy, Poromechanics, England: J. Wiley \& Sons, 2004.

[48] A. V. Neimark, P. I. Ravikovitch and A. Vishnyakov, "Bridging scales from molecular simulations to classical thermodynamics: density functional theory of capillary condensation in nanopores," J. Phys.: Condens. Matter, vol. 15, pp. 347-365, 2003. 
[49] R. Pelton, "Poly(N-isopropylacrylamide) (PNIPAM) is never hydrophobic," Journal of Colloid and Interface Science, vol. 348, pp. 673-674, 2010.

[50] J. J. Liétor-Santos and A. Fernández-Nieves, "Motion of microgels in electric fields," Advances in colloid and interface science, vol. 147, pp. 178-185, 2008.

[51] J. Zhang and R. Pelton, "The dynamic behavior of poly(N-isopropylacrylamide) at the air/water interface," Colloids and Surfaces, vol. 156, pp. 111-122, 1999. 